\title{
论文
}

\section{考虑多种污染物时空分布的电力系统高维多目标 优化调度策略}

\author{
余涛", 陈艺璇, 张孝顺 \\ 华南理工大学电力学院, 广州 510640 \\ *E-mail: taoyu1@scut.edu.cn
}

收稿日期: 2017-10-17; 接受日期: 2018-03-20; 网络版发表日期: 2018-06-19

国家重点基础研究发展计划(编号: 2013CB228205)和国家自然科学基金(批准号: 51477055, 51777078)资助项目

摘要 提出了一种全新的考虑多种污染物时空分布的电力系统高维多目标优化调度策略. 首先, 构建出一种真正 适用于电力调度的火电厂污染物时空分布模型, 首次将大气边界层的日变化考虑在内, 充分体现了火电厂的污染 物扩散特征, 提升了结果的准确性; 然后, 结合各类污染物的时空分布特点及环境容量, 建立同时减少发电成本、 碳排放及 $\mathrm{PM}_{2.5}, \mathrm{SO}_{2}, \mathrm{NO}_{2}$ 空气质量影响的高维多目标优化调度模型; 最后, 借助具有代表性的高维多目标优化算 法获得近似的帕累托最优解集, 并提出一种考虑目标特征与环境容量的多目标决策方法篮选折中解. 采用自建的 模拟城市案例以及广东省案例进行仿真, 结果表明: 所述调度方法不仅可以有效改善空气质量, 还可根据环境容 量的时空变化做出相应的调整, 真正意义上实现经济、环保的电力调度.

关键词大气边界层, 火电厂污染物时空分布模型, 高维多目标优化, 电力调度, 环境容量

\section{1 引言}

全球变暖与大气污染是威胁人类生存的两大环境 问题. 火电厂在发电过程中会产生大量的 $\mathrm{CO}_{2}$ 及大气 污染物，其中 $\mathrm{PM}_{2.5}, \mathrm{SO}_{2}, \mathrm{NO}_{2}$ 的影响最为严重. 近年 来, 国内外学者进行了很多关于低碳、低大气污染电 力系统的研究, 主要包括: 排放后处理技术 ${ }^{[1]}$ 、清洁新 能源发电技术 ${ }^{[2 \sim 4]}$ 、考虑环境、经济因素的电力调度 技术 ${ }^{[5 \sim 12]}$ 等. 其中，环境经济调度(environmental/economic dispatch, EED)因成本低、见效快而越来越受到 广泛关注.
早期EED的研究多将污染物的排放量作为经济目 标的约束 ${ }^{[5]}$ 或利用价格罚因子 ${ }^{[6]}$ 将排放量嵌入经济目 标函数, 从而将多目标问题转化为单目标问题来求解. 然而, 这种方法无法很好地体现经济目标与环境目标 之间的折中关系，且排放量约束限值、排放价格罚因 子值的合理性也很难被保证; 多目标EED通过同时优 化经济、环境目标，有效避免了上述问题，成为近年 来的研究热点. 文献[7]将火电厂排放的所有类型的污 染物一起考虑, 建立起多目标EED模型, 并提出改进的 非支配排序遗传算法(non-dominated sorting genetic algorithm, NSGA)进行求解. 针对上述模型, 文献[8,9]

引用格式: 余涛, 陈艺璇, 张孝顺. 考虑多种污染物时空分布的电力系统高维多目标优化调度策略. 中国科学: 技术科学, 2018, 48: 755-772 Yu T, Chen Y X, Zhang X S. Many-objective optimization dispatching strategy for power system considering the temporal and spatial distribution of different pollutants (in Chinese). Sci Sin Tech, 2018, 48: 755-772, doi: 10.1360/N092017-00355 
又进一步提出了多目标粒子群算法、改进的细菌受食 算法以求得收敛性、均匀性更优的帕累托前沿(Pareto front，PF). 文献[10,11]进一步考虑了机组的多种燃料 来源、阀点效应及禁止运行区间，建立了非凸的多目 标EED模型，并提出自组织分层粒子群算法、多目标 蚁狮算法进行求解. 文献[12]根据不同污染物的排放 特性，将 $\mathrm{NO}_{x}$ 和 $\mathrm{SO}_{2}$ 分开考虑，建立了考虑多种污染物 排放的多目标EED模型，并采用改进的交互式多目标 优化算法进行求解.

然而，上述关于EED的研究都是从排放量的角度 对发电产生的 $\mathrm{CO}_{2}, \mathrm{PM}_{2.5}, \mathrm{SO}_{2}, \mathrm{NO}_{2}$ 进行控制. 对于以 $\mathrm{CO}_{2}$ 为主的温室气体而言, 其在大气中的含量直接决 定着大气对地表发射的长波辐射的吸收能力以及对地 表的加热能力, 因此从排放量的角度对 $\mathrm{CO}_{2}$ 进行控制 确实能够有效缓解全球变暖 ${ }^{[13]}$. 然而, 对于 $\mathrm{PM}_{2.5}, \mathrm{SO}_{2}$, $\mathrm{NO}_{2}$ 这类大气污染物而言, 衡量其危害程度的真正标 准应为地面浓度 (ground level concentration, GLC)而 非排放量. 2007年, 我国正式启动覆盖全国的空气质量 监测网建设工作 ${ }^{[14]}$, 对包括 $\mathrm{PM}_{2.5}, \mathrm{SO}_{2}, \mathrm{NO}_{2}$ 在内的多种 大气污染物的GLC进行实时监测, 并于2012年正式采 用空气质量指数(air quality index, AQI)标准对各监测 点的 GLC进行评级 ${ }^{[15]}$, 以衡量各污染物对人体健康的 影响. 可见，从 $\mathrm{GLC}$ 的角度对火电厂产生的大气污染 物进行控制才是更直接有效的方法. GLC虽受到排放 量的影响，但也在很大程度上取决于污染源的高度、 位置以及风速、风向、温度、大气边界层(atmospheric boundary layer, ABL) 高度、大气稳定度等气象 条件. 不同的气象条件下, 污染物的稀释、扩散特性将 呈现出很大差异，即使排放量相同，GLC也会截然 不同.

目前，从 GLC的角度对火电厂大气污染物进行控 制的电力调度研究非常少见, 并且尚未真正提出一种 适用于电力调度的火电厂污染物时空分布模型. 文献 [16]所采用的基本高斯烟羽扩散模型虽计算简便，但 却认为气象参数在污染物扩散过程中是固定不变的, 在气象条件随时空迅速变化的情况下计算结果十分不 准确. 并且, 对于极易出现严重大气污染事故的小静风 天气(平均风速 $\leq 1 \mathrm{~m} / \mathrm{s}$ ), 高斯烟羽扩散模型完全失 效 ${ }^{[17]}$; 相比而言, 文献[18]所采取的基本高斯烟团扩散 模型可以应用于小静风天气情况，也可以体现气象条 件的时空变化. 但是, 此模型没有考虑ABL的日变化
对污染物扩散的影响，并不适用于火电厂这类高架点 源 ${ }^{[19]}$, 且忽略了污染物因各种清除转化机制造成的质 量损失, 计算误差仍较大.

准确有效的火电厂污染物时空分布模型的缺失导 致相关部门目前仍然是从排放量的角度对火电厂进行 管控, 所依据的排放标准通常都是基于较差情况做出 的保守估计. 但其实大气环境本身具有一定的自净能 力(环境容量), 当气象条件不利于污染物的扩散与稀 释、环境容量小时，虽严格的排放量控制法并不一定 是最有效的途径, 但也可以在一定程度上缓解大气污 染. 可是, 当环境容量大时, 不考虑污染物的时空分布 特性、忽视环境本身的自净能力、继续一味地进行排 放限制将直接导致火电厂因购买低污染的清洁煤或不 得不更换现有设备而徒增成本. 因此, 亟需一种比排放 量限制法更有效的调度策略来降低火电厂的环境影 响, 并能够灵活适应环境容量的时空变化, 实现经济与 环保的有机统一.

为了解决上述问题，本文提出一种全新的调度策 略. 首先构建出一种真正适合于电力调度的火电厂污 染物时空分布模型, 然后建立起考虑多种污染物时空 分布的电力系统高维多目标优化调度模型, 并提出一 种考虑目标特征与环境容量的多目标决策方法来选择 合适的折中解. 最后, 在自建的模拟城市和广东省两个 案例上进行仿真, 证明所述调度策略的有效性、优势 及实用价值.

\section{2 火电厂污染物时空分布模型}

适用于电力调度的火电厂污染物时空分布模型必 须满足以下条件：1）将ABL的影响考虑在内, 充分体 现火电厂这类高架点源的污染物扩散特征;2) 适用于 各种气象条件, 能体现大气中各种清除转化机制带来 的质量损失, 结果足够准确; 3) 计算简便, 不会造成电 力调度的运算负担. 首先对基本高斯烟团模型、 $\mathrm{ABL}$ 的日变化及其影响进行介绍, 然后建立起满足上述要 求的火电厂污染物时空分布模型.

\section{1 基本高斯烟团模型}

基本高斯烟团模型采用一系列分离的烟团来模拟 从烟图口排放出的连续烟流, 如图1所示. 首先根据计 算精度要求、平均风速等确定基本时步 $\Delta t_{\mathrm{b}}$, 基本时段 


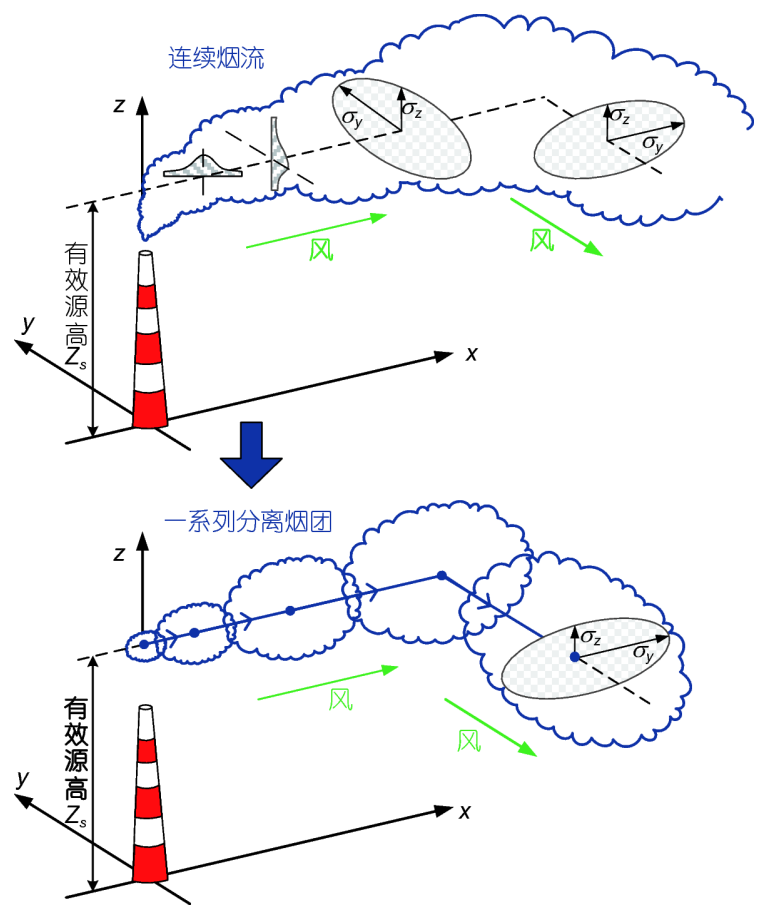

图 1 (网络版彩图)基本高斯烟团模型原理说明图

$i:\left[t_{i-1}, t_{i}\right]$ 满足: $t_{i}=t_{i-1}+\Delta t_{\mathrm{b} i}$. 污染源在每个基本时段之初 排放出一个烟团，时段内的排放强度、风速、风向、 大气稳定度都认为是恒定的. 某污染源在空间某点某 时刻造成的GLC等于在此之前排放的所有烟团的GLC 的叠加. 空气质量监测站每隔 $\Delta t_{\mathrm{b}}$ 进行一次浓度监测, 则第 $k$ 个时段结束时, 位置 $(x, y)$ 处的 GLC可以计算为 ${ }^{[18]}$

$C\left(t_{k} ; x, y\right)=\sum_{i=1}^{k} M(i) G\left(t_{i-1}, t_{k} ; x, y\right)$,

式中, $t_{i-1}$ 表示时段 $i$ 的烟团排放时刻, $M(i)$ 表示时段 $i$ 的 烟团质量, $G(\cdot)$ 为一个单元烟团的污染物时空分布函 数. 假定每个烟团的物质浓度在水平和垂直方向上呈 高斯分布, 则 $G\left(t_{i-1}, t_{k} ; x, y\right)$ 满足:

$$
\left\{\begin{array}{l}
G\left(t_{i-1}, t_{k} ; x, y\right)=\frac{\exp \left[-\left(\Delta_{y}+\Delta_{z}\right) / 2\right]}{(2 \pi)^{3 / 2} \sigma_{y}^{2}\left(t_{i-1}, t_{k}\right) \sigma_{z}\left(t_{i-1}, t_{k}\right)}, \\
\Delta_{y}=\frac{\left[x_{\mathrm{c}}\left(t_{i-1}, t_{k}\right)-x\right]^{2}+\left[y_{\mathrm{c}}\left(t_{i-1}, t_{k}\right)-y\right]^{2}}{\sigma_{y}^{2}\left(t_{i-1}, t_{k}\right)}, \\
\Delta_{z}=z_{\mathrm{c}}^{2}\left(t_{i-1}, t_{k}\right) / \sigma_{z}^{2}\left(t_{i-1}, t_{k}\right),
\end{array}\right.
$$

式中, $\left[x_{\mathrm{c}}, y_{\mathrm{c}}, z_{\mathrm{c}}\right]$ 为烟团中心位置坐标, $\sigma_{y}$ 为水平(包括 $x$ 和 $y$ 两个方向)扩散参数, $\sigma_{z}$ 为垂直扩散系数 $(z$ 方向), 表 征烟团扩散的范围. 烟团中心位置受风的支配而不断
改变, 如图1所示, 可按下式计算:

$$
\left[\begin{array}{l}
x_{c}\left(t_{i-1}, t_{k}\right) \\
y_{c}\left(t_{i-1}, t_{k}\right) \\
z_{c}\left(t_{i-1}, t_{k}\right)
\end{array}\right]=\left[\begin{array}{l}
x_{s} \\
y_{s} \\
z_{s}
\end{array}\right]+\sum_{k^{\prime}=i}^{k}\left[\begin{array}{l}
u\left(k^{\prime}\right) \\
v\left(k^{\prime}\right) \\
w\left(k^{\prime}\right)
\end{array}\right] \cdot \Delta t_{\mathrm{b} k^{\prime}},
$$

式中, $u\left(k^{\prime}\right), v\left(k^{\prime}\right), w\left(k^{\prime}\right)$ 分别为第 $k^{\prime}$ 个时段内 $x, y, z$ 方向上 的平均风速, $\left(x_{s}, y_{s}\right)$ 和 $z_{s}$ 分别为污染源的水平位置坐标 和有效源高.

$\sigma_{y}, \sigma_{z}$ 取决于烟团的运行时间和大气对污染物的扩 散和稀释能力, 在烟团排放的时段中, 有:

$\left\{\begin{array}{l}\sigma_{y}\left(t_{i-1}, t\right)=\alpha(i)\left(t-t_{i-1}\right)^{a(i)}, \\ \sigma_{z}\left(t_{i-1}, t\right)=\beta(i)\left(t-t_{i-1}\right)^{b(i)},\end{array}\right.$

式中, $\alpha, \beta, a, b$ 为扩散参数计算系数, 取决于时段烟团 所处大气层结的稳定度等级, 根据风速、云量、太阳 高度角等被划分为 $1 \sim 6$, 越小代表越不稳定， 4 代表中 性, 具体见文献[20]. 各时段气象要素的变化使其扩散 能力各不相同, 两相邻时段的扩散参数应满足烟团扩 散廓线的连续性, 计算为 ${ }^{[20]}$

$$
\left\{\begin{array}{l}
\sigma_{y}\left(t_{i-1}, t_{k}\right)=\left[\alpha(k)^{1 / a(k)} \Delta t_{\mathrm{b} k}+\sigma_{y}\left(t_{i-1}, t_{k-1}\right)^{1 / a(k)}\right]^{a(k)}, \\
\sigma_{z}\left(t_{i-1}, t_{k}\right)=\left[\beta(k)^{1 / b(k)} \Delta t_{\mathrm{b} k}+\sigma_{z}\left(t_{i-1}, t_{k-1}\right)^{1 / b(k)}\right]^{b(k)} .
\end{array}\right.
$$

\section{$2.2 \mathrm{ABL}$ 的日变化及其影响}

\subsection{1 $\mathrm{ABL}$ 的概念及其日变化特征}

$\mathrm{ABL}$ 位于地球表面和自由大气之间，是与人类活 动关系最直接、最密切的底层大气. ABL内部气流的 水平运动称为风, 支配着污染物的水平输送; 不规则 运动称为湍流，决定着污染物在垂直方向上的迁移与 扩散 ${ }^{[19]}$. 通常把白天的ABL主要分为混合层(mixed layer， ML)、卷夹层(entrainment layer，EL); 把夜间 ABL主要分为稳定边界层(stable boundary layer, SBL)、剩余层(residual layer, RL), 典型的日变化是 $\mathrm{ABL}$ 的基本特征 ${ }^{[21]}$, 如图2所示.

日出之后，地面获得的太阳辐射能以感热和潜热 的形式向上输送, 加热大气, 使得不稳定层结的ML从 地表开始发展，原本在夜间形成的SBL将自下而上逐 渐消散, 发展为EL存在于ML的顶部，随着ML一同上 升. 地面供热为ML中的大气湍流提供能量, 有利于其 内部的污染物在垂直方向上的混合与扩散; EL为稳定 


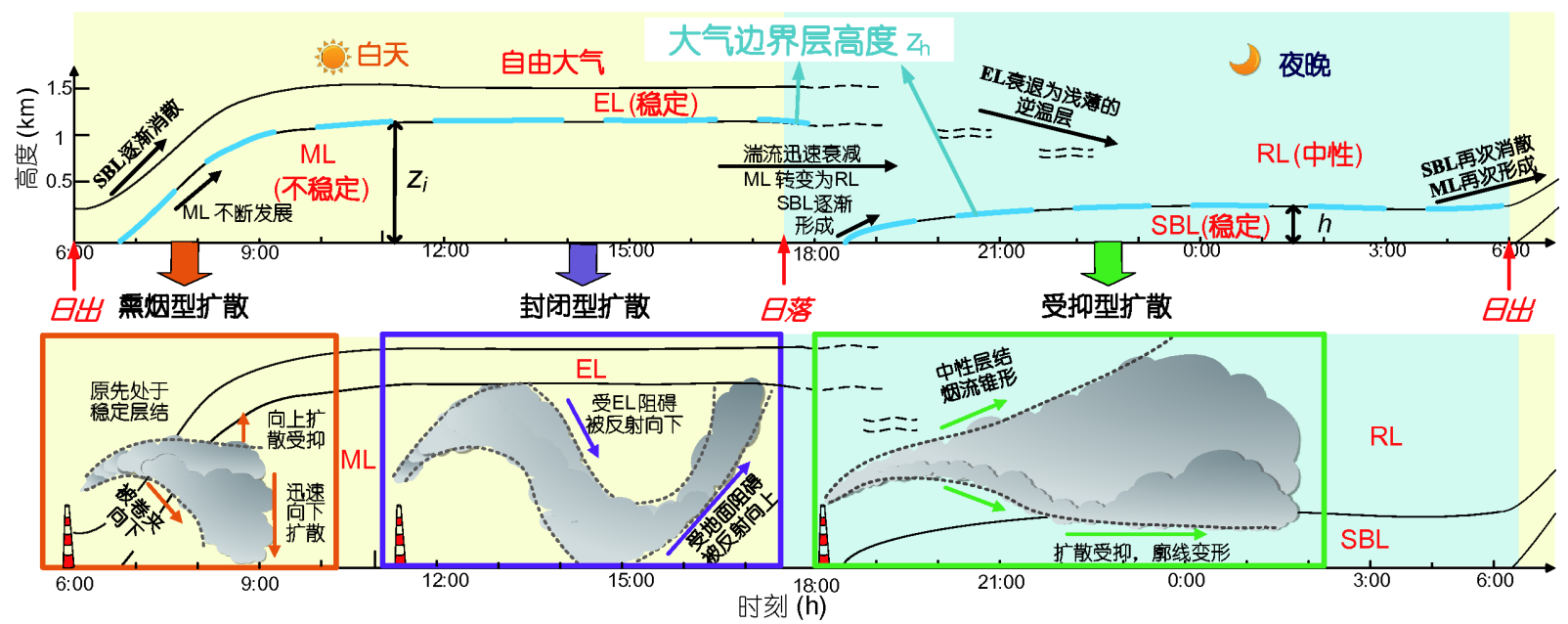

图 2 (网络版彩图)ABL的日变化特征及相应的扩散形式

层结, 内部湍流非常弱, 故像盖子一样将污染物阻碍在 ML内. ML在热力驱动下不断发展, 于午后达到最大高 度(1 2 km).

日落后, 地表冷却导致ABL从下往上降温, 逐渐发 展成为层结稳定的SBL; 原先ML中的湍流由于热量损 失而强烈衰减，最终转变成中性层结的RL存在于 SBL 上方; 原先的EL也将逐渐减弱变成一个或多个浅薄的 逆温层存在于半空. SBL内部的湍流能量很弱, 故其最 大高度比日间ML低的多, 通常至100 500 m. ABL高度 由ML高度 $z_{i}$ 和SBL高度 $h$ 共同定义 ${ }^{[22]}$, 记作 $z_{h}$, 即:

$$
z_{h}=\left\{\begin{array}{l}
z_{i} \text { (白天) }, \\
h \text { (夜晚) } .
\end{array}\right.
$$

\subsubsection{ABL 影响下的火电厂污染物扩散形式}

火电厂污染物以烟流的形式从烟图口排出，有效 源高通常在 $500 \mathrm{~m}$ 左右 ${ }^{[23]}$, 属于典型的高架点源, 其扩 散受到ABL的日变化尤其是 $z_{h}$ 变化的强烈影响，呈现 出3种典型的形式: 熏烟型扩散、封闭型扩散、受抑 型扩散, 如图2所示.

熏烟型扩散 ${ }^{[19]}$ 多发生于清晨，与日出后SBL消 散、ML形成的时间相吻合，针对的是原本排放在稳 定大气层结中的污染物, 如夜间排放的污染物, 或ML 高度尚小于火电厂有效源高时, 排放在ML上方的污染 物。一旦ML发展，这些因垂直扩散受抑而大量积聚在
稳定层结中的污染物将被逐渐卷夹进ML. 由于向上扩 散受到上方稳定层结的抑制, 被卷夹的污染物将在ML 强湍流的支配下迅速向下扩散，几乎瞬间在垂直方向 均匀分布. 熏烟型扩散虽持续时间较短, 但往往造成 地面高浓度, 后果可能比较严重.

封闭型扩散 ${ }^{[24]}$ 发生在白天的大部分时间、ML高 度较高时, 针对的是直接排放进ML的污染物. 烟流上 缘到达ML顶部时, 因受到EL阻碍而无法继续向上, 将 在湍流的作用下重新混合向下; 同样地, 烟流下缘将因 地面阻碍而无法继续向下，在湍流的作用下重新混合 向上. 烟流整体被封闭在EL和地面之间, 故称为封闭 型扩散. 当不考虑EL的穿透和地面对污染物的吸附作 用时, 污染物就如同被封闭在两个完全反射面之间, 不 断地被扩散、反射、再扩散直至无限, 如图2所示. 封 闭型扩散的污染物本身就排放在不稳定层结中，不存 在积聚过程, 故对GLC的影响通常没有熏烟型扩散那 么严重, 但持续时间较长.

受抑型扩散 ${ }^{[25]}$ 主要发生在夜晚、SBL形成之后. SBL内部的稳定大气对湍流起到抑制作用，既不利于 其内部污染物的稀释, 也不利于RL中的污染物扩散向 下； RL为相当厚度的中性层结，湍流强度在各个方向 上几乎相同. 通常, 夜晚SBL高度小于火电厂的有效源 高, 污染物直接排放在RL内, 并在RL中以几乎相等的 速率向水平、垂直方向扩散, 烟流廓线呈雉形. 当烟流 的下缘伸达SBL顶部时，廓线将因垂直扩散受抑而变 形, 如图2所示. 


\section{3 火电厂污染物时空分布模型}

\subsection{1 连续烟流的精确模拟}

取每个电力调度时段为一基本时段 $\Delta t_{\mathrm{b}}$, 同样地, 每个 $\Delta t_{\mathrm{b}}$ 内电厂出力及各类气象条件保持恒定. 但是, 一个调度时段通常为 $15 \mathrm{~min} \sim 1 \mathrm{~h}$, 若仍令每个 $\Delta t_{\mathrm{b}}$ 只释 放一个烟团，则两相邻烟团之间的距离可能较远，不 能很好地模拟连续烟流. 因此，本文将烟团释放时步 $\Delta t_{\mathrm{p}}$ 缩短为 $\Delta t_{\mathrm{b}}$ 的 $1 / N_{\mathrm{p}}, \Delta t_{\mathrm{p}}$ 应满足任意风速下相邻烟团间 的距离都小于烟团半宽 ${ }^{[26]}$, 即:

$\left[u^{2}(i)+v^{2}(i)\right]^{1 / 2} \cdot \Delta t_{\mathrm{p}} \leq R_{\mathrm{p} y}, i=1,2, \ldots, N$,

式中, $N$ 为一天的调度时段总数, $R_{\mathrm{p} y}$ 为烟团半宽, 定义 为: $R_{\mathrm{p} y}=2.15 \sigma_{y}{ }^{[19]}$. 相应地, 式(3)和(4)更新为

$$
\begin{aligned}
& {\left[\begin{array}{l}
x_{\mathrm{c}}\left(\tau, t_{k}\right) \\
y_{\mathrm{c}}\left(\tau, t_{k}\right) \\
z_{\mathrm{c}}\left(\tau, t_{k}\right)
\end{array}\right]=\left[\begin{array}{l}
x_{s} \\
y_{s} \\
z_{s}
\end{array}\right]+\left[\begin{array}{l}
u\left(k_{\tau}\right) \\
v\left(k_{\tau}\right) \\
w\left(k_{\tau}\right)
\end{array}\right]\left(t_{k_{\tau}}-\tau\right)+\sum_{k^{\prime}=k_{\tau}+1}^{k}\left[\begin{array}{l}
u\left(k^{\prime}\right) \\
v\left(k^{\prime}\right) \\
w\left(k^{\prime}\right)
\end{array}\right] \Delta t_{\mathrm{b} k^{\prime}},} \\
& \left\{\begin{array}{l}
\sigma_{y}(\tau, t)=\alpha\left(k_{\tau}\right)(t-\tau)^{a\left(k_{\tau}\right)} \\
\sigma_{z}(\tau, t)=\beta\left(k_{\tau}\right)(t-\tau)^{b\left(k_{\tau}\right)}
\end{array}\right.
\end{aligned}
$$

式中， $\tau$ 表示烟团释放的时刻， $k_{\tau}$ 表示时刻 $\tau$ 所属的基本 时段, $t_{k \tau}$ 表示时段 $k_{\tau}$ 的结束时刻.

\subsection{2 考虑 $\mathrm{ABL}$ 日变化的烟团扩散}

基本高斯烟团模型没有考虑ABL的影响，认为烟 才浓度在水平、垂直方向上始终呈高斯分布. 但实际 上, $\mathrm{ABL}$ 高度 $z_{h}$ 上下的稳定度等级差别很大, 火电厂污 染物烟团的浓度并不完全呈高斯形式. 现用 $z_{h}(k)$ 表示 时段 $k$ 的ABL高度、 $S(k)$ 表示该时段 $z_{h}(k)$ 高度以下大气 层结的稳定度等级，仍以火电厂第 $k_{\tau}$ 个时段内 $\tau$ 时刻排 放的烟团为例，分析熏烟型、封闭型、受抑型3种形 式下的时空分布函数 $G(\cdot)$.

1) 若烟团以熏烟形式扩散, 条件为

$\left[S\left(k_{\tau}\right) \geq 4 \mid z_{s}>z_{h}\left(k_{\tau}\right)\right] \& S(k)<4$,

式中, $S\left(k_{\tau}\right) \geq 4$ 表示 $k_{\tau}$ 时段 $\mathrm{ABL}$ 为夜间下方 $\mathrm{SBL}$ 、上方 RL的结构, 此时, 烟团排放于RL或SBL内; $z_{s}>z_{h}\left(k_{\tau}\right)$ 表 示夜晚或白天 $z_{h}$ 较低、烟团排放在SBL或ML上方的情 况，这两种情况下的污染物原先都处于稳定层结. $S(k)$ $<4$ 表示监测时段 $k$ 中ML已经发展，将逐步把原处于稳 定层结的污染物卷夹向下，进而形成熏烟型扩散. 通
常假设物质浓度在原稳定层结中呈高斯分布，被卷夹 的部分在ML垂直方向上均匀分布、水平方向上仍呈 高斯分布, 则 $G(\cdot)$ 为 ${ }^{[19]}$

$$
\left\{\begin{array}{l}
G\left(\tau, t_{k} ; x, y\right)=\frac{\Phi(p) \cdot \exp \left(-\Delta_{y F} / 2\right)}{2 \pi \sigma_{y F}^{2}\left(\tau, t_{k}\right) z_{h}(k)}, \\
\Delta_{y F}=\left\{\left[x_{\mathrm{c}}\left(\tau, t_{k}\right)-x\right]^{2}+\left[y_{\mathrm{c}}\left(\tau, t_{k}\right)-y\right]^{2}\right\} / \sigma_{y F}^{2}\left(\tau, t_{k}\right), \\
\Phi(p)=\int_{-\infty}^{p} \frac{1}{\sqrt{2 \pi}} \exp \left(-\frac{1}{2} p^{2}\right) \mathrm{d} p, \\
p\left(t_{k}\right)=\left[z_{h}(k)-z_{\mathrm{c}}\left(\tau, t_{k}\right)\right] / \sigma_{z 0}\left(\tau, t_{k}\right), \\
\sigma_{y F}\left(\tau, t_{k}\right)=\sigma_{y 0}\left(\tau, t_{k}\right)+z_{\mathrm{c}}\left(\tau, t_{k}\right) / 8,
\end{array}\right.
$$

式中, $\Phi(p)$ 表示被卷夹进入ML的烟团质量占烟团总质 量的比例, $\Phi(p) \in[0,1] . p$ 为表征烟团进入ML程度的参 数. $\sigma_{y 0}$ 和 $\sigma_{z 0}$ 表示烟团原先处于稳定层结时的水平、垂 直扩散系数; $\sigma_{y F}$ 表示进入ML之后的水平扩散系数, 比 $\sigma_{y 0}$ 多一个扩展修正项.

2) 若烟团呈现封闭型扩散, 条件为

$S\left(k_{\tau}\right)<4 \& z_{s} \leq z_{h}\left(k_{\tau}\right) \& S(k)<4$,

式中, $S\left(k_{\tau}\right)<4 \quad \& \quad z_{s} \leq z_{h}\left(k_{\tau}\right)$ 表示 $k_{\tau}$ 时段ML已经发展且超 过污染源的有效源高，烟团直接排放进ML内. $S(k)<4$ 表示 $k$ 时段 $\mathrm{BBL}$ 仍为下方 ML、上方EL的结构，直接排 放至ML的烟团将被封闭在EL和地面之间，扩散呈现 出“两个反射面”的特点, 浓度分布可采用像源法 ${ }^{[19,24]}$ 计算, 推导过程见文献[19], 若不考虑污染物对EL的 穿透及地面对污染物的吸附, 可得到:

$$
\left\{\begin{aligned}
G\left(\tau, t_{k} ; x, y\right)= & \frac{\exp \left(-\Delta_{y} / 2\right) \cdot R\left(\tau, t_{k}\right)}{(2 \pi)^{3 / 2} \sigma_{y}^{2}\left(\tau, t_{k}\right) \sigma_{z}\left(\tau, t_{k}\right)}, \\
R\left(\tau, t_{k}\right)= & \sum_{n=-N_{R}}^{N_{R}} \exp \left[-\left(2 n z_{h}(k)-z_{\mathrm{c}}\left(\tau, t_{k}\right)\right)^{2} / 2 \sigma_{z}^{2}\left(\tau, t_{k}\right)\right](13) \\
& +\sum_{n=-N_{R}}^{N_{R}} \exp \left[-\left(2 n z_{h}(k)+z_{\mathrm{c}}\left(\tau, t_{k}\right)\right)^{2} / 2 \sigma_{z}^{2}\left(\tau, t_{k}\right)\right],
\end{aligned}\right.
$$

式中, $R(\cdot)$ 为按像源法求得的反射项, $n$ 表示反射次数, $N_{R}$ 为误差允许范围内的最大反射计算次数, 通常 $N_{R}=4$ 即可满足要求 ${ }^{[20]}$. $\Delta_{y}$ 的定义同式(2).

3) 若烟团呈现受抑型扩散, 条件为

$S(k) \geq 4$.

此时，监测时段 $k$ 已形成夜间 SBL，其上方为中性 的RL. 污染物进入SBL后扩散受抑所发生的廓线变形 
其实是烟团扩散范围即扩散参数 $\sigma_{y}$ 和 $\sigma_{z}$ 的变化, 可以通 过改变式(5)中 $\alpha, \beta, a, b$ 的值来实现, 物质浓度分布在 垂直和水平方向上仍呈高斯形式, 即 $G(\cdot)$ 为

$$
G\left(\tau, t_{k} ; x, y\right)=\frac{\exp \left[-\left(\Delta_{y}+\Delta_{z}\right) / 2\right]}{(2 \pi)^{3 / 2} \sigma_{y}^{2}\left(\tau, t_{k}\right) \sigma_{z}\left(\tau, t_{k}\right)},
$$

式中, $\Delta_{y}$ 和 $\Delta_{z}$ 的定义同式(2), 反映烟团扩散受抑的扩散 参数计算系数求取方法如下式所示:

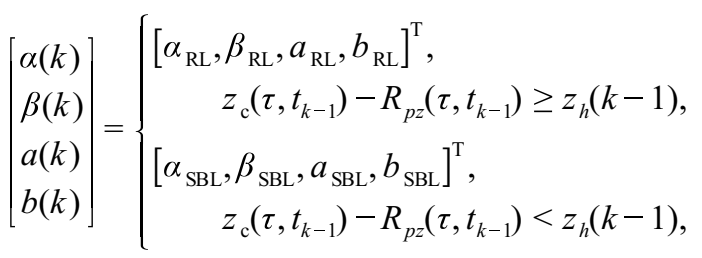

式中， $R_{\mathrm{p} z}$ 为烟团半高，定义为 $R_{\mathrm{p} z}=2.15 \sigma_{z}^{[19]} ; z_{\mathrm{c}}\left(\tau, t_{k-1}\right)$ $-R_{\mathrm{p} z}\left(\tau, t_{k-1}\right)$ 表征第 $k-1$ 个时段结束时的烟团下缘位置, 若低于 $z_{h}(k-1)$, 则证明烟团在时段 $k-1$ 内进入了 $\mathrm{SBL}$, 下一时段 $k$ 扩散受抑; 否则, 时段 $k$ 应仍按RL的湍流特 性向水平、垂直方向扩散.

\subsection{3 污染物的影响寿命}

基本高斯烟团模式假定污染物在大气中是保守 的, 不因任何物理、化学过程而发生转化, 也不被清 除 ${ }^{[18]}$. 但实际上, 污染物在输送扩散过程中存在着多 种清除转化机制，包括：由重力沉降以及被土壤、植 物、建筑物等吸附而引起的干沉积、由雨水冲刷等带 来的湿沉积、化学转化等. 这些机制都会引起污染物 的质量损失，进而影响GLC. 通常的处理方法是假定 污染物质量随时间呈指数衰减 ${ }^{[27]}$, 则时段 $k$ 结束时, 一 个单元烟团造成的GLC为

$c\left(\tau, t_{k} ; x, y\right)=M(\tau) \cdot \exp \left[-\frac{t_{k}-\tau}{T_{\text {res }}}\right] \cdot G\left(\tau, t_{k} ; x, y\right)$,

式中, $T_{\text {res }}$ 为污染物的居留期(residence time $)^{[19]}$, 表征污 染物的影响寿命, 其值取决于污染物特性、地点、气 象条件等, 短至几小时, 长则几天, 综合反映了大气中 各种清除转化机制的作用.

\subsubsection{GLC的计算}

同样地， $t_{k}$ 时刻 $(x, y)$ 处的 GLC 为在此之前排放的 所有烟团的GLC的叠加，综合考虑了连续烟流的精确 模拟、ABL日变化及污染物影响寿命后, 计算为

$$
\left\{\begin{array}{c}
C\left(t_{k} ; x, y\right)=\sum_{\tau=0}^{t_{k}-\Delta t_{\mathrm{p}}} M(\tau) \cdot \exp \left[-\frac{t_{k}-\tau}{T_{\mathrm{res}}}\right] \cdot G\left(\tau, t_{k} ; x, y\right), \\
G\left(\tau, t_{k} ; x, y\right) \\
=\left\{\begin{array}{r}
{\left[2 \pi \sigma_{y F}^{2}\left(\tau, t_{k}\right) z_{h}(k)\right]^{-1} \cdot \Phi(p) \cdot \exp \left(-\Delta_{y F} / 2\right),} \\
{\left[S\left(k_{\tau}\right) \geq 4 \mid z_{s}>z_{h}\left(k_{\tau}\right)\right] \& S(k)<4,} \\
{\left[(2 \pi)^{3 / 2} \sigma_{y}^{2}\left(\tau, t_{k}\right) \sigma_{z}\left(\tau, t_{k}\right)\right]^{-1} \cdot \exp \left(-\Delta_{y} / 2\right) \cdot R\left(\tau, t_{k}\right),} \\
S\left(k_{\tau}\right)<4 \& z_{s} \leq z_{h}\left(k_{\tau}\right) \& S(k)<4, \\
{\left[(2 \pi)^{3 / 2} \sigma_{y}^{2}\left(\tau, t_{k}\right) \sigma_{z}\left(\tau, t_{k}\right)\right]^{-1} \cdot \exp \left[-\left(\Delta_{y}+\Delta_{z}\right) / 2\right],} \\
S(k) \geq 4 .
\end{array}\right.
\end{array}\right.
$$

\section{3 考虑多种污染物时空分布的电力系统高 维多目标优化调度模型}

\section{1 高维多目标优化问题}

多目标优化问题(multi-objective optimization problem, MOP)通常可以描述为

$$
\begin{cases}\min & F(\mathbf{x})=\left(f_{1}(\mathbf{x}), f_{2}(\mathbf{x}), \ldots, f_{M}(\mathbf{x})\right), \\
\text { s.t. } & \left\{\begin{array}{l}
g(\mathbf{x})=0, \\
h(\mathbf{x}) \leq 0
\end{array}\right.\end{cases}
$$

式中, $\mathbf{x}$ 为决策向量, $M$ 为子目标个数, $g(\mathbf{x})$ 为等式约束, $h(\mathbf{x})$ 为不等式约束. 对于可行解 $\mathbf{x}^{*}$, 若不存在比 $\mathbf{x}^{*}$ 所有 目标值都小的其他可行解, 则称 $\mathbf{x}$ *⿻一为非劣解. 整个决策 空间内的非劣解集合称为帕累托最优解集, 对应的目 标向量集称为PF. 特别地, 当 $M \geq 4$ 时, 将此MOP称为高 维多目标优化问题(many-objective optimization problem, MaOP). 因求解难度和复杂度远高于两目标、 三目标优化问题, MaOP已经成为MOP中一个独立的 分支, 越来越受到优化领域的广泛关注.

\section{2 目标函数}

目标1：最小化发电成本(generation cost, GC). 假 定一个电厂只有一种类型的机组, 且出力平均分配. 在 实际中, 机组通常具有多种燃料来源, 电厂会在不同的 出力区间选择不同的燃料以满足经济性. 此外, 当机组 在不同出力区间、处于不同的运行状态时, 其能耗特 性也不同. 这都将使机组的发电成本特性在不同出力 区间内发生变化. 为使模型更符合工程实际, 本文采 
用由两段或多段函数组成的机组发电成本函数，表示 为 ${ }^{[28-31]}$

$$
\left\{\begin{aligned}
\min \mathrm{GC}_{k_{\tau}} & =\sum_{i=1}^{N_{\mathrm{G}}} \mathrm{GC}_{i}\left(P_{i k_{\tau}}\right) \cdot N_{\mathrm{u} i}, \\
\mathrm{GC}_{i}\left(P_{i k_{\tau}}\right)= & \left\{\begin{array}{c}
a_{i 1}+b_{i 1} P_{i k_{\tau}}+c_{i 1} P_{i k_{\tau}}^{2}, P_{i}^{\min } \leq P_{i k_{\tau}} \leq P_{i 1}, \\
a_{i 2}+b_{i 2} P_{i k_{\tau}}+c_{i 2 h} P_{i k_{\tau}}^{2}, P_{i 1}<P_{i k_{\tau}} \leq P_{i 2}, \\
\vdots \\
a_{i h}+b_{i h} P_{i k_{\tau}}+c_{i h} P_{i k_{\tau}}^{2}, P_{i(h-1)}<P_{i k_{\tau}} \leq P_{i}^{\max },
\end{array}\right.
\end{aligned}\right.
$$

式中, $\mathrm{GC}_{k \tau}$ 为时段 $k_{\tau}$ 的总发电成本, $P_{i k_{\tau}}$ 表示电厂 $i$ 的一台 机组在时段 $k_{\tau}$ 的有功出力, $P_{i}^{\min }$ 及 $P_{i}^{\max }$ 分别为电厂 $i$ 机组 的出力上下限. $\mathrm{GC}_{i}\left(P_{i k_{\tau}}\right)$ 为 $i$ 电厂机组的发电成本函数, $a_{i h}, b_{i h}, c_{i h}$ 为 $i$ 电厂机组在出力区间 $h$ 内的发电成本系数. $N_{\mathrm{G}}$ 为某区域内电厂总数, $N_{\mathrm{u}}$ 为电厂 $i$ 的机组个数.

目标2: 最小化 $\mathrm{CO}_{2}$ 排放量 (emission of $\left.\mathrm{CO}_{2}, \mathrm{EC}\right)$. 不同种类燃料的单位热值含碳量不同，且当机组处于 不同的出力区间和运行状态时，能耗特性及排放特性 也会发生较大的变化，故 $\mathrm{CO}_{2}$ 排放方程也为分段函数, 即 $^{[32]}$ :

$$
\left\{\begin{aligned}
& \min \mathrm{EC}_{k_{\tau}}= \sum_{i=1}^{N_{\mathrm{G}}} \mathrm{EC}_{i}\left(P_{i k_{\tau}}\right) \cdot N_{\mathrm{ui}}, \\
& \mathrm{EC}_{i}\left(P_{i k_{\tau}}\right)=\left\{\begin{array}{c}
d_{i 1}+e_{i 1} P_{i k_{\tau}}+f_{i 1} P_{i k_{\tau}}^{2}, P_{i}^{\mathrm{min}} \leq P_{i k_{\tau}} \leq P_{i 1}, \\
d_{i 2}+e_{i 2} P_{i k_{\tau}}+f_{i 2 h} P_{i k_{\tau}}^{2}, P_{i 1}<P_{i k_{\tau}} \leq P_{i 2}, \\
\vdots \\
d_{i h}+e_{i h} P_{i k_{\tau}}+f_{i h} P_{i k_{\tau}}^{2}, P_{i(h-1)}<P_{i k_{\tau}} \leq P_{i}^{\max },
\end{array}\right.
\end{aligned}\right.
$$

式中, $\mathrm{EC}_{k \tau}$ 表示所有电厂在 $k_{\tau}$ 时段内排放的 $\mathrm{CO}_{2}$ 总量, $\mathrm{EC}_{i}\left(P_{i k_{\tau}}\right)$ 为 $i$ 电厂机组的 $\mathrm{CO}_{2}$ 排放特性方程, $d_{i h}, e_{i h}, f_{i h}$ 为 $i$ 电厂机组在出力区间 $h$ 中的 $\mathrm{CO}_{2}$ 排放系数.

考虑到同一火电厂 $\mathrm{PM}_{2.5}, \mathrm{SO}_{2}, \mathrm{NO}_{2}$ 的排放强度差 异、不同污染物在大气中的传播特性差异及环境容量 差异, 将 $\mathrm{PM}_{2.5}, \mathrm{SO}_{2}, \mathrm{NO}_{2}$ 分开、同时优化, 形成目标 3 5, 即: 最小化火电厂排放的 $\mathrm{PM}_{2.5}, \mathrm{SO}_{2}, \mathrm{NO}_{2}$ 的空气质 量影响(effects on air quality, EAQ). EAQ定义为在考虑 污染物时空分布与环境容量空间差异的情况下，火电 厂排污在整个居留期内对某一特定区域空气质量的影 响大小, 其值等于对该区域内所有监测点EAQ的均值.

由式(18)知， $G(\cdot)$ 只受地理位置、气象条件的影 响, 与火电厂出力无关. 因此在地理位置和气象条件 已知的情况下, 电厂污染物的时空分布系数为一常数: $\mu_{i j k_{\tau} k}^{A P}=\sum_{\tau=t_{k_{\tau}}-1}^{t_{k_{\tau}}-\Delta t_{\mathrm{p}}} \exp \left[-\frac{t_{k}-\tau}{T_{\mathrm{res}}^{A P}}\right] \cdot G\left(\tau, t_{k} ; x_{j}, y_{j}\right)$,

式中, 上标 $A P$ 表示 $\mathrm{PM}_{2.5}, \mathrm{SO}_{2}, \mathrm{NO}_{2}$ 中某一特定的污染 物, $\mu_{i j k_{\tau} k^{A}}$ 为电厂 $i$ 在 $k_{\tau}$ 时段产生的污染物 $A P$ 在时段 $k$ 结束 时刻于监测点 $j$ 处的时空分布系数, 等于在 $k_{\tau}$ 时段内排 放的所有烟团的浓度分布的叠加. $T_{\text {res }}^{A P}$ 为某一特定污 染物的居留期.

各监测点的气象条件、背景浓度不同，其所处大 气环境对火电厂污染物的容量也不同, 环境容量小的 监测点将对火电厂排污更加敏感. 本文借助各监测点 各污染物的空气质量分指数(individual air quality index, IAQI)级别来评估当地环境对某污染物的容量大 小. IAQI对应的污染级别越高, 该时段该污染物的环 境容量越小, 则电厂在该时段对该监测点的EAQ越大. 同样地，在地理位置和气象条件已知的情况下，电厂 $i$ 在时段 $k_{\tau}$ 排放的污染物对一特定区域的 $\mathrm{EAQ}$ 系数 $\pi_{i k_{\tau}}{ }_{\tau}$ 为一常数, 可计算为

$$
\pi_{i k_{\tau}}^{A P}=\frac{1}{N_{\mathrm{m}}} \sum_{j=1}^{N_{\mathrm{m}}} \sum_{k=k_{\tau}}^{k_{\tau}+k_{\text {res }}^{A P}-1} \mu_{i j k_{\imath} k}^{A P} \cdot \eta\left(k ; x_{j}, y_{j}\right),
$$

式中, $N_{\mathrm{m}}$ 为该区域监测点总个数, $\eta\left(k ; x_{i}, y_{i}\right)$ 为时段 $k$ 监 测点 $\left(x_{j}, y_{j}\right)$ 处的环境容量罚系数, 求取方法如表 1 所示. $k_{\mathrm{res}}^{A P}$ 为某一特定污染物的居留期所覆盖的基本时段个 数, 即 $k_{\mathrm{res}}^{A P}=T_{\mathrm{res}}^{A P} / \Delta t_{\mathrm{b}}$. 由上式可知, $\pi_{i k_{\tau}}^{A P}$ 表征了 $k_{\tau}$ 时段排 污在未来整个居留期内造成的影响, 并通过 $\eta$ 考虑了环 境容量的空间差异. 显然, $\mu$ 与 $\pi$ 均为与机组出力无关的 常数, 在应用于电力调度时不会造成计算负担. 综上所 述, 目标3 5可以表示为

$$
\left\{\begin{array}{l}
\min \operatorname{EAQ}_{k_{\tau}}^{A P}=\sum_{i=1}^{N_{\mathrm{G}}} \operatorname{EAQ}_{i}^{A P}\left(P_{i k_{\tau}}\right), \\
\mathrm{EAQ}_{i}^{A P}\left(P_{i k_{\tau}}\right)=\frac{1}{N_{\mathrm{p}}} \cdot N_{\mathrm{ui}} \cdot M_{i}^{A P}\left(P_{i k_{\tau}}\right) \cdot \pi_{i k_{\tau}}^{A P}, \\
M_{i}\left(P_{i k_{\tau}}\right)=\left\{\begin{array}{c}
g_{i 1}^{A P}+m_{i 1}^{A P} P_{i k_{\tau}}+l_{i 1}^{A P} P_{i k_{\tau}}^{2}, P_{i}^{\min } \leq P_{i k_{\tau}} \leq P_{i 1}, \\
g_{i 2}^{A P}+m_{i 2}^{A P} P_{i k_{\tau}}+l_{i 2}^{A P} P_{i k_{\tau}}^{2}, P_{i 1}<P_{i k_{\tau}} \leq P_{i 2}, \\
\vdots \\
g_{i h}^{A P}+m_{i h}^{A P} P_{i k_{\tau}}+l_{i h}^{A P} P_{i k_{\tau}}^{2}, P_{i(h-1)}<P_{i k_{\tau}} \leq P_{i}^{\max },
\end{array}\right.
\end{array}\right.
$$

式中, $\mathrm{EAQ}^{A P}{ }_{k \tau}$ 表示所有电厂在 $k_{\tau}$ 时段内排放的污染物 
表 1 根据环境容量确定 $\eta$ 与 $W_{i}^{\text {p.u }}$

\begin{tabular}{ccccc}
\hline 空气质量指数级别 ${ }^{[15]}$ & $\operatorname{IAQI}^{A P}\left(k ; x_{j}, y_{j}\right)$ & $\eta^{A P}\left(k ; x_{j}, y_{j}\right)$ & $\operatorname{AIAQI}^{A P}\left(k_{\tau}\right)$ & $W_{i}^{\text {p.u }}(i=3,4,5)$ \\
\hline 优 & $0 \sim 50$ & 0.5 & $0 \sim 50$ & 0.5 \\
良 & $51 \sim 100$ & 1 & $51 \sim 100$ & 1 \\
轻度污染 & $101 \sim 150$ & 2 & $101 \sim 150$ & 2 \\
中度污染 & $151 \sim 200$ & 3 & $151 \sim 200$ & 3 \\
重度污染 & $201 \sim 300$ & 4 & $201 \sim 300$ & 4 \\
严重污染 & $>300$ & 5 & $>300$ & 5 \\
\hline
\end{tabular}

的空气质量影响, $\mathrm{EAQ}^{A P}{ }_{i}$ 为电厂 $i$ 的空气质量影响. $M_{i}^{A P}$ $\left(P_{i k_{\tau}}\right)$ 为 $i$ 电厂一台机组在 $k_{\tau}$ 时段产生的污染物质量, 假 定同一个电厂机组的污染物从同一个烟图排出, 则每 个烟团的质量为 $\left(N_{\mathrm{u}} / N_{\mathrm{p}}\right) \cdot M_{i}^{A P}$; 不同种类燃料的灰分、 硫分等不同，且机组在不同出力下的能耗特性、燃烧 方式也会发生变化，故排污特性方程同样也为分段函 数, $g_{i h}{ }^{A P}, m_{i h}{ }^{A P}, l_{i h}{ }^{A P}$ 为 $i$ 电厂机组在出力区间 $h$ 中的大气 污染物 $A P$ 的排污系数.

综上所述，本文所述的考虑多种污染物时空分布 的电力系统高维多目标优化调度模型的目标函数为

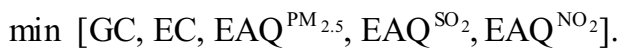

\section{3 约束条件}

搭建的电力调度模型只关注有功的调度，且不考 虑网络约束. 因此, 式(25)需同时满足容量上下限约 束、有功功率平衡约束以及爬坡约束:

$$
\left\{\begin{array}{l}
P_{i}^{\min } \leq P_{i k_{\tau}} \leq P_{i}^{\max }, i=1,2, \ldots, N_{\mathrm{G}}, \\
\sum_{i=1}^{N_{\mathrm{G}}} P_{i k_{\tau}} \cdot N_{\mathrm{u} i}-P_{D k_{\tau}}=0, \\
-D R_{i} \leq P_{i k_{\tau}}-P_{i\left(k_{\tau}-1\right)} \leq U R_{i}, i=1,2, \ldots, N_{\mathrm{G}},
\end{array}\right.
$$

式中, $P_{D k_{\tau}}$ 为 $k_{\tau}$ 时段的有功总负荷. $D R_{i}$ 和 $U R_{i}$ 分别为电 厂 $i$ 机组的下降爬坡速率和上升爬坡速率限值.

\section{4 求解算法及决策机制}

\section{1 高维多目标优化算法概述}

$\mathrm{MaOP}$ 的目标个数较多, 非劣解规模及计算复杂 度较大, $\mathrm{PF}$ 的不连续、非凸等特征也比二、三目标的

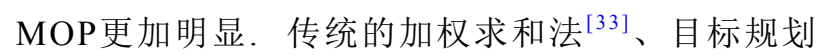
法 $^{[34]} 、 \varepsilon-$ 约束法 ${ }^{[34]}$ 等常采用目标权重、目标期望值、 上限 $\varepsilon$ 值等将MOP转化为单目标问题. 但是, 随着目标 个数的增多, 决策者在参数设置时需要处理的数据也 会相应变多，参数设置的合理性将很难被保证；并且， 传统优化方法需要多次运行单目标优化才能获取PF, 随着目标个数的增多, 试验次数将呈指数增长, 求解效 率将大幅下降. 再加上传统方法对PF的形状较为敏感, 很难胜任非凸 $\mathrm{MaOP}$ 的求解, 故并不适用于本文所述 模型.

目前绝大多数MaOP优化算法都集中在智能优化 领域, 经典的多目标进化算法(multi-objective evolutionary algorithm, MOEA)如NSGA, NSGA-II, 强度帕 累托进化算法(strength Pareto evolutionary algorithm, SPEA)虽在解决二目标或三目标优化问题时表现出优 越的性能. 但受到 $\mathrm{MaOP}$ 中目标个数增多、非劣解规 模增大的影响, 其选择与搜索能力被严重削弱, 性能 大幅下降 ${ }^{[35]}$. 目前, 研究者已经提出多种改进的 MOEA以应对 MaOP, 主要包括以下几类：1）仍基于 Pareto支配进行排序, 但结合其他技术来降低目标维 度或控制种群在目标空间中的分布, 如NSGA-III ${ }^{[36]}$; 2）基于松散Pareto支配进行排序，如格支配法 ${ }^{[37]}$; 3) 非Pareto支配排序, 如性能评价指标进化算法 ${ }^{[38]}$ (indicator-based evolutionary algorithm, IBEA)、基于分解 的多目标进化算法 ${ }^{[39]}$ (multi-objective evolutionary algorithm based on decomposition, MOEA/D)等. 研究表明, 上述算法在求解 $\mathrm{MaOP}$ 时均能够产生收敛性、分布性 以及延展性优越的Pareto最优解集, 且能够很好地逼近 不连续或非凸的PF, 故均可应用于求解本文所构建的 考虑多种污染物时空分布的高维多目标优化调度 模型. 


\section{2 考虑目标特征与环境容量的多目标决策}

为了既能客观反映各目标间的冲突性与相关性, 又能根据气象条件的变化对调度计划做出灵活调整, 本文采用考虑目标特征与环境容量的逼近理想解排序 法(technique for order preference by similarity to ideal solution, TOPSIS)从帕累托解集中选择合适的折中解, 为调度人员提供决策信息. 主要包括规格化处理、组 合赋权和TOPSIS决策 3 个步骤.

\subsection{1 规格化处理}

由于目标函数之间存在着量纲和数量级的差异, 故对原始数据进行规格化处理, 如下所示:

$b_{i j}=\frac{f_{i}^{\max }-f_{i}\left(\mathbf{x}_{j}\right)}{f_{i}^{\max }-f_{i}^{\min }}, i=1,2, \ldots, M, j=1,2, \ldots, N$,

式中, $M$ 为目标个数, $N$ 为非劣解总个数. $f_{i}\left(\mathbf{x}_{j}\right)$ 为非劣解 $j$ 对应的目标 $i$ 函数值, $f_{i}^{\max }$ 和 $f_{i}^{\min }$ 分别为所有非劣解目标 $i$ 函数值的最大值和最小值, 由此得到规范化的决策矩 阵 $\mathbf{B}=\left(b_{i j}\right)_{\mathrm{M} \times \mathrm{N}}$. 显然, $b_{i j}$ 越大, $f_{i}\left(\mathbf{x}_{j}\right)$ 越优.

\subsection{2 组合赋权}

1) 确定反映目标特征的客观权重向量 $\mathbf{W}^{\mathrm{obj}}$. 本文 的 5 个目标之间存在着冲突, 但目标 $3 \sim 5$ 之间又呈现出 一定的相关性. 如使用优质燃料时各类污染物的排污 系数可能均较小、静小风时各类污染物的 $\mathrm{EAQ}$ 可能 均较大等. 因此, 采用指标相关性权重分析法(criteria importance through intercriteria correlation, CRITIC) ${ }^{[40]}$ 制定客观权重, 如下式所示:

$W_{i}^{\mathrm{obj}}=C_{i} / \sum_{i=1}^{M} C_{i}, C_{i}=\sigma_{i} \sum_{k=1}^{M}\left(1-r_{i k}\right)$,

式中, $C_{i}$ 为目标 $i$ 所包含的信息量, 信息量越大权重越 大. $\sigma_{i}$ 为 $\mathbf{B}$ 矩阵第 $i$ 行的标准差, 反映各非劣解在目标 $i$ 上的函数值差距, 差距越大信息量越大; $r_{i k}$ 为 $\mathbf{B}$ 矩阵第 $i$ 行和第 $k$ 行的相关系数, 反映目标 $i, k$ 之间的相关性. 目 标 $i$ 与其余目标间的相关性越大, 信息量就越少.

2) 确定体现环境容量变化的主观权重向量 $\boldsymbol{W}^{\mathrm{sub}}$. 随着气象条件的不断变化, 大气环境对火电厂在不同 时段排放出的污染物的容量也在变化. 本文根据 $k_{\tau}$ 时 段未来居留期内的IAQI均值 $\mathrm{AIAQI}^{A P}\left(k_{\tau}\right)$ 来衡量环境对 $k_{r}$ 时段排放的污染物 $A P$ 的容量, 计算为
$\operatorname{AIAQI}^{A P}\left(k_{\tau}\right)=\frac{1}{k_{\mathrm{res}}^{A P}} \sum_{k=k_{\tau}}^{k_{\tau}+k_{\mathrm{res}}^{A P}-1} \operatorname{IAQI}^{A P}(k)$.

取目标 1,2 的主观权重标么值 $W_{i}^{\mathrm{p} . \mathrm{u}}(i=1,2)$ 为 1 , 由 AIAQI ${ }^{A P}$ 确定 $W_{i}^{\text {p.u. }}(i=3,4,5)$ 的方法如表1所示. 由表可 知, $\mathrm{AIAQI}^{A P}\left(k_{\tau}\right)$ 越大, 环境对 $k_{\tau}$ 时段污染物 $A P$ 的容量越 小, 主观上对 $\mathrm{EAQ}^{A P}$ 的重视程度越大; 反之, 弱化了对 $\mathrm{EAQ}^{A P}$ 的重视, 从而更好地改善其他环境容量较小的 大气污染物的EAQ, 或更强调策略的经济性和低碳性. 最终, 各目标的主观权重为

$W_{i}^{\text {sub }}=W_{i}^{\text {p.u. }} / \sum_{i=1}^{5} W_{i}^{\text {p.u. }}$.

3) 根据主客观权重得到最优组合权重向量 $\mathbf{W}^{*}$. 一 方面, $\mathbf{W}^{*}$ 应与 $\mathbf{W}^{\mathrm{obj}}, \mathbf{W}^{\mathrm{sub}}$ 之间的偏差应尽量小, 以较好 地反映主客观权重的信息; 另一方面, $\mathbf{W}^{*}$ 应满足使整 个帕累托解集的综合评价值尽可能大. 故构建如下所 示的最优化模型, 求得 $\mathbf{W}^{*[41]}$ :

$\begin{cases}\min & \sum_{i=1}^{M}\left[\left(W_{i}^{*}-W_{i}^{\mathrm{obj}}\right)^{2}+\left(W_{i}^{*}-W_{i}^{\mathrm{sub}}\right)^{2}\right]-\sum_{i=1}^{M} \sum_{j=1}^{N} b_{i j} W_{i}^{*}, \\ \text { s.t. } & \sum_{i=1}^{M} W_{i}^{*}=1,0 \leq W_{i}^{*} \leq 1 .\end{cases}$

式(31)为一单目标非线性规划问题, 采用经典的 非线性规划法 ${ }^{[42]}$ 即可求解, 最终得到 $\mathbf{W}^{*}$.

\subsubsection{TOPSIS决策}

TOPSIS法是一种有效的多目标决策方法, 首先根 据B矩阵及最优组合权重 $\mathbf{W}^{*}$, 得到加权标准化决策矩 阵, 并进一步确定正理想点和负理想点; 然后根据各 个解与正负理想点的距离进行排序, 满足距离正理想 点最近、同时距离负理想点距离最远的解即为折中 解, 具体计算及步骤见文献[43].

\subsection{4 求解步骤}

综上所述, 本文所提出的考虑多种污染物时空分 布的电力系统高维多目标优化调度策略的执行步骤如 图3所示. 可以看出, 电网只需在原有电力调度计算的 基础上增加火电厂污染物时空分布计算过程, 即可实 现考虑污染物时空分布的电力调度. 所得到的 $\pi$ 为与 机组出力无关的常数, 不会在调度计划优化阶段造成 额外的计算负担. 每个时段的优化开始之前, 需要对 


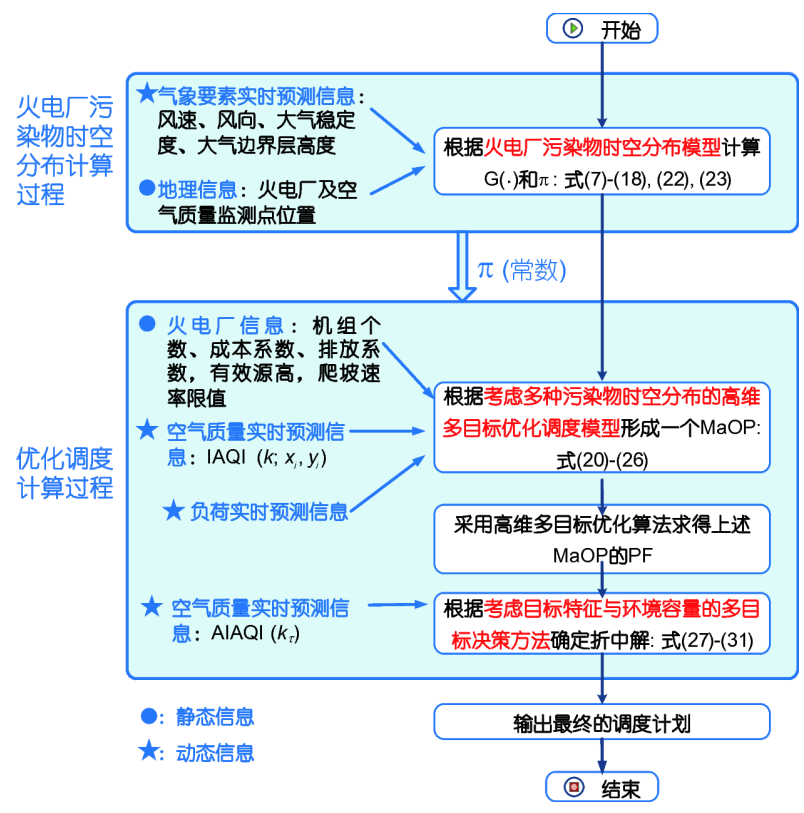

图 3 (网络版彩图)考虑多种污染物时空分布的电力系统高 维多目标优化调度策略执行流程

动态信息进行更新, 其中, 气象要素信息、空气质量信 息为从中央气象台信息发布系统(http://www.nmc.cn/ publish/forecast/china.html)、全国空气质量预报信息 发布系统(http://106.37.208.228:8082/)所获取的实时预 报信息，其余信息的获取方式与传统EED模型的方式 相同.

\section{5 算例仿真}

根据大气污染时空分布考量尺度的不同，将仿真 分为单个城市尺度和含多个城市的区域尺度两个案例 展开. 首先以自建的模拟城市为例, 并考虑污染日和非 污染日两个典型场景，验证本文所述电力调度策略的 有效性及优势; 然后以广东省为例，根据实际气象条 件及负荷情况制定调度计划，通过对比分析证明本文 所述调度策略的实用价值.

\section{1 对比调度策略及求解方法设计}

根据各污染物的典型特性, 所有案例中 $\mathrm{PM}_{2.5}, \mathrm{SO}_{2}$, $\mathrm{NO}_{2}$ 的居留期均取 $22,24,20 \mathrm{~h}^{[44]}$, 调度时段 $\Delta t_{\mathrm{b}}$ 均为 $1 \mathrm{~h}$, $N_{\mathrm{p}}$ 均取 60 , 即 $\Delta t_{\mathrm{p}}=1 \mathrm{~min}$. 均采用 4 种策略制定次日调度 计划: 策略 1 , 只考虑发电成最小化的单目标电力调度; 策略 2 , 在追求发电成本最小化的同时, 也最小化电厂
所产生的 $\mathrm{CO}_{2}$ 及各类大气污染物的排放量；策略 3 , 除 最小化发电成本、 $\mathrm{CO}_{2}$ 排放量外，还从 GLC的角度对 大气污染物控制，但只考虑一种污染物 $\left(\mathrm{PM}_{2.5}\right)$ 的影响; 策略4, 本文提出的考虑多种污染物时空分布的电力系 统高维多目标优化调度. 4种调度策略的目标函数如表 2所示, 其中, $\mathrm{E}^{A P}$ 表示当前调度时段污染物 $A P$ 的排 放量.

策略 1 是一个非线性、不连续且不可微的单目标 优化问题，考虑到传统优化算法求解此类问题时的局 限性, 且为避免单一算法的片面性, 本文选取遗 传 ${ }^{[4]}$ 、粒子群 ${ }^{[4]}$ 、群搜索 ${ }^{[46]} 3$ 种启发式算法进行求解, 并将仿真进行 10 次, 在 30 个结果中选出成本最小的解 作为策略1的调度计划.

策略2 策略 4 是目标函数不连续、不可微的MOP, 其中策略 2 和 4 为 $\mathrm{MaOP}$. 为避免单一算法的片面性, 本 文从4.1节所述的多种MOEA中挑选出具有代表性的 三种: NSGA-III ${ }^{[36]}$, IBEA ${ }^{[38]}, \mathrm{MOEA} / \mathrm{D}^{[39]}$ 进行求解, 并 将仿真进行10次. 采用反世代距离(inverted generation distance, IGD)对所获得的 30 组近似帕累托解集进行评 价 ${ }^{[47]}$, 选取IGD值最小的一组作为最优近似帕累托解 集. 在多目标决策方法上, 策略2采用的排放量限制法 不考虑环境容量, 故采用CRITIC法得到的客观权重为 各目标赋权; 策略 3 和 4 从 GLC 的角度对大气污染物进 行控制，故可根据AIAQI将环境容量考虑在内，采用 4.2.2所述组合赋权法确定各目标权重. 最后, 基于上 述权重用TOPSIS法在最优近似帕累托解集中篮选出 折中解, 作为各策略的调度计划.

\section{2 火电厂污染物时空分布计算过程耗时分析}

如前所述, $\pi$ 为与机组出力无关的常数, 故策略 4 中 的调度计划优化过程与传统EED优化过程的计算复杂 度、计算耗时无异. 但策略4在优化调度过程之前增加 了火电厂污染物时空分布计算过程，为验证其是否能 够满足电网实时优化调度的运行需求，本文对策略4 中火电厂污染物时空分布计算过程进行了耗时分析. 由式(7) (18), (22)和(23)可知, $\pi$ 的维度及计算复杂度 受到火电厂个数 $N_{\mathrm{G}}$ 、空气质量监测点个数 $N_{\mathrm{m}} 、 \mathrm{GLC}$ 监测时步 $\Delta t_{\mathrm{b}}$ 、烟团排放时步 $\Delta t_{\mathrm{p}}$ 、各类污染物的居留 时间 $T_{\mathrm{res}}{ }^{A P}$ 的大小的影响. 模拟城市案例及广东省案例 的参数设置及其火电厂污染物时空分布计算过程的平 均耗时如表3所示, 可以看出, 即使对于整个广东省而 
表 2 仿真算例对比调度策略设计

\begin{tabular}{|c|c|c|}
\hline 调度策略 & 目标个数 & 目标函数 \\
\hline 策略1 & 1 & $\min \mathrm{GC}$ \\
\hline 策略2 & 5 & $\min \left[\mathrm{GC}, \mathrm{EC}, \mathrm{E}^{\mathrm{PM}_{2.5}}, \mathrm{E}^{\mathrm{SO}_{2}}, \mathrm{E}^{\mathrm{NO}_{2}}\right]$ \\
\hline 策略3 & 3 & $\min \left[\mathrm{GC}, \mathrm{EC}, \mathrm{EAQ}^{\mathrm{PM}_{2.5}}\right]$ \\
\hline 策略4 & 5 & 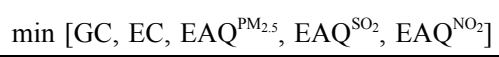 \\
\hline
\end{tabular}

表 3 火电厂污染物时空分布计算过程耗时分析

\begin{tabular}{|c|c|c|c|c|c|c|c|c|}
\hline \multirow{2}{*}{ 仿真案例 } & \multicolumn{7}{|c|}{ 参数设置 } & \multirow{2}{*}{$\begin{array}{l}\text { 火电厂污染物时空分算过程平均耗时 } \\
\text { 布计算 }\end{array}$} \\
\hline & $N_{\mathrm{G}}$ & $N_{\mathrm{m}}$ & $\Delta t_{\mathrm{b}}$ & $\Delta t_{\mathrm{p}}$ & $T_{\text {res }}{ }^{\mathrm{PM}_{2.5}}$ & $T_{\text {res }} \mathrm{SO}_{2}$ & $T_{\mathrm{res}} \mathrm{NO}_{2}$ & \\
\hline 模拟城市案例 & 10 个 & 5 个 & $1 \mathrm{~h}$ & $1 \mathrm{~min}$ & $22 \mathrm{~h}$ & $24 \mathrm{~h}$ & $20 \mathrm{~h}$ & $0.32 \mathrm{~s}$ \\
\hline 广东省案例 & 59 个 & 104 个 & $1 \mathrm{~h}$ & $1 \mathrm{~min}$ & $22 \mathrm{~h}$ & $24 \mathrm{~h}$ & $20 \mathrm{~h}$ & $3.28 \mathrm{~s}$ \\
\hline
\end{tabular}

言，计算出其内 59 家火电厂在下一时段排污的所有 $\mathrm{EAQ}$ 系数 $\pi$ 也仅需 $3.28 \mathrm{~s}$, 充分证明了本文所提出的火 电厂污染物时空分布模型不会对原有电力调度带来太 多时延, 完全可以满足电网实时优化调度的运行要求.

\section{3 模拟城市案例}

此案例中每个电厂只有一类机组, 各机组间出力 均匀分配. 电厂与空气质量监测站的地理位置如图4 所示, 机组的出力区间、燃料类型、发电成本系数见 文献[28], 其余信息如附录表S1所示.

本文在模拟城市案例中选取了气象条件不利于污 染物扩散、且电厂处于城市上风向的污染日场景, 及 气象条件有利于污染物扩散、且电厂处于城市下风向 的非污染日场景进行对比仿真, 以充分说明气象条件 对大气污染物GLC的影响, 并证明从时空分布的角度 来实施火电厂大气污染控制的必要性. 为使仿真具有 一定可比性，两个场景的负荷曲线被设置为完全相同， 如图5所示.

\subsection{1 污染日场景}

污染日气象数据见附录表 $\mathrm{S} 2$, 其特征为: 整个城市 受静小风控制(风速 $\leq 1 \mathrm{~m} / \mathrm{s}), z_{h}$ 较低( $\leq 835 \mathrm{~m}$ ), 且大气 层结偏向于稳定, 不利于污染物的扩散、稀释与输送, 预测IAQI的污染等级偏高. 且当日该城市主导风为东 北风转东南风, 大部分电厂都处于城市的上风向, 对 城市空气质量的影响较大.

取所有监测点的浓度均值作为该城市的浓度 ${ }^{[14]}, 4$ 种策略下该城市的 $\mathrm{PM}_{2.5}, \mathrm{SO}_{2}, \mathrm{NO}_{2}$ 实时GLC及 $\mathrm{ABL}$ 实

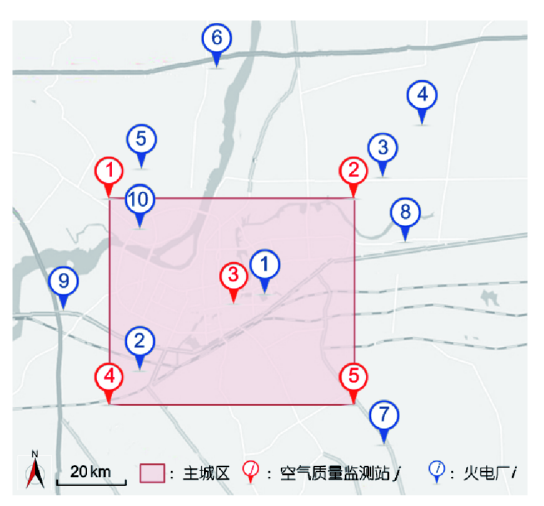

图 4 (网络版彩图)模拟城市案例地理位置分布图

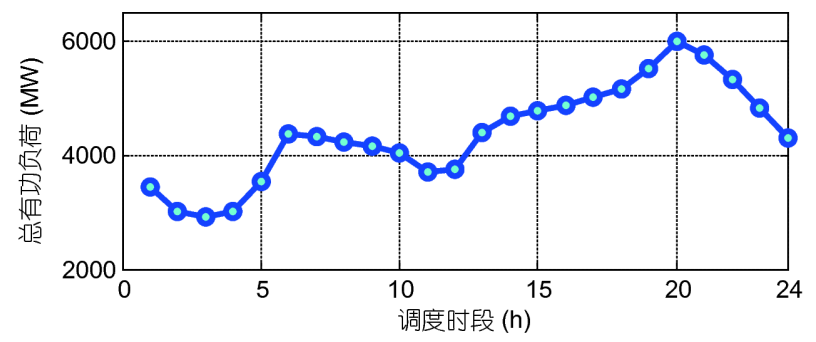

图 5 (网络版彩图)模拟城市案例污染日与非污染日场景负 荷曲线

时高度如图6所示, 其中背景色块表示该城市各时段各 类污染物的预测IAQI等级. 可以看出, 各类污染物的 GLC 与ABL的日变化呈现出明显的相关性: 当ML逐 渐发展但低于火电厂有效源高时, 污染物以熏烟形式 造成地面高浓度(8:00 11:00); 随着ML的不断升高, 污 染物将直接排放于ML内并呈现封闭型扩散, GLC比熏 烟型扩散时降低(12:00 16:00); 夜间SBL逐渐形成后, 


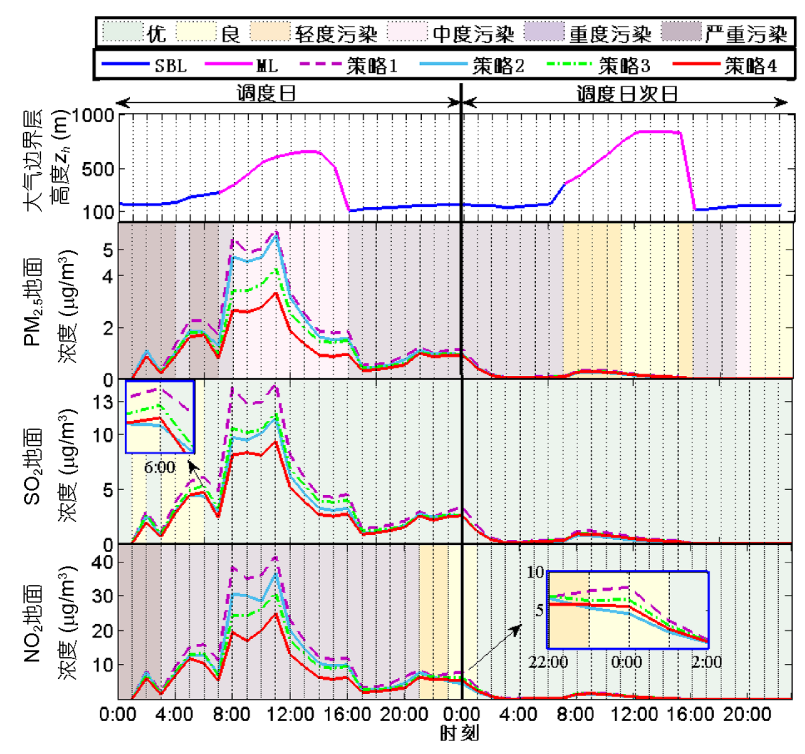

图 6 污染日场景ABL实时高度及各策略实时GLC

污染物向下扩散受抑, GLC比白天明显降低 (17:00 20:00). 进入SBL的污染物需经较长的时间才 能扩散到地，形成GLC的小幅度上升(21:00 次日 0:00)；调度日污染物的影响会持续至调度日次日，当 SBL消散、ML再次形成时, 污染物再次以熏烟形式使 GLC上升(次日 8:00 10:00). 污染物质量在各种清除转 化机制的作用下不断减少, 次日GLC明显小于当日, 并 在居留期后期逐渐趋近于 0 .

调度日 10:00的 $\mathrm{PM}_{2.5}, \mathrm{SO}_{2}, \mathrm{NO}_{2}$ 的GLC详细示于如 图7. 结合图6和7可以看出, 策略2虽控制了污染物的排 放量，但对GLC的优化结果并不理想; 策略3虽降低了 $\mathrm{PM}_{2.5}$ 的 GLC, 但 $\mathrm{SO}_{2}, \mathrm{NO}_{2}$ 的 GLC 仍处于一个偏高的值, 尤其是 10:00时各监测点 $\mathrm{NO}_{2}$ 的预测IAQI达到重度污 染以上等级，仅偏重于一种污染物的优化显然是不够 合理的；而策略4的调度计划可同时大幅减少 3 种污染 物的GLC. 此外，当环境容量较小时，策略4的优化力 度明显加大, 如PM 2.5 的GLC几乎在各时刻较其他策略 都明显减少， $\mathrm{NO}_{2}$ 的 GLC 在0:00 21:00期间明显减少 等; 而当环境容量较大时，策略4会弱化对该污染物的 控制力度, 如 $\mathrm{SO}_{2}$ 在 $6: 00$ 时的 $G L C$ 及 $\mathrm{NO}_{2}$ 在次日 $0: 00$ 时 的GLC会比其他策略小幅上升.

4种策略的调度计划如图8所示. 可以看出: (1) 与 策略1相比, 策略4中电厂 $1,2,4$ 在 0:00 14:00期间的出 力明显减少, 几乎达到其出力的最低限值. 这是因为这 段时间内城市盛行东北风, 这 3 个电厂处于城市的上风

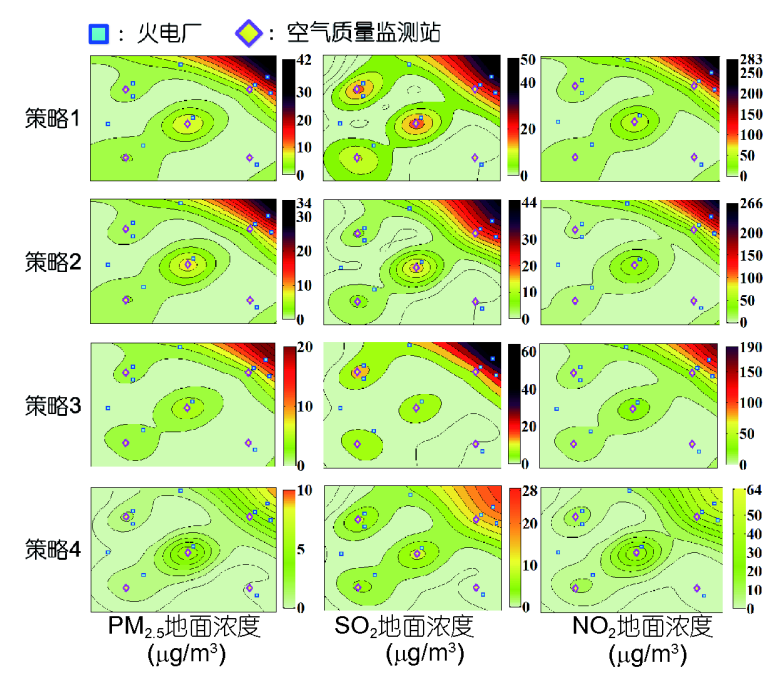

图 7 污染日场景10:00各策略地面浓度

向, 所产生的污染物将对城市空气质量产生较大影响; 而在14:00之后，电厂1，2，4对空气质量的影响随着风 向的转变而逐渐减小, 机组出力逐渐上升; (2) 与策略 2 相比, 策略 4 中电厂 6,9 的出力明显上升, 这是因为这两 个电厂的污染物排放系数虽大，但其所处位置对城市 空气质量影响小, 尤其是电厂9, 因其全天都处于城市 的下风向而几乎 $24 \mathrm{~h}$ 满发; (3) 虽策略3和 4 都是从 GLC 的角度对大气污染物进行控制，但图中黄色圆点和红 色曲线却存在大量不一致. 充分说明了由于排放强 度、扩散特性、环境容量差异，大气污染物之间并非 线性相关，只考虑一种污染物很有可能导致对电厂机 组出力的限制不够(如对电厂 2,4 )或过度限制(如对电 厂5).

4种策略的调度结果如表4所示，其中TGC和TEC 表示 24 个时段的发电总成本(total generation cost, $\mathrm{TGC}$ )和 $\mathrm{CO}_{2}$ 总排放量(total emission of $\mathrm{CO}_{2}, \mathrm{TEC}$ ); TE 表示 24 个时段大气污染物的总排放量(total emission, TE), TGLC和AGLC分别表示 24 个时段的排污在其寿 命内各时刻的GLC总值(total ground level concentration, TGLC)和每小时平均GLC值(average ground level concentration, AGLC). 即:

$$
\left\{\begin{aligned}
\operatorname{TGLC}^{A P}= & \sum_{k=1}^{24+k_{\text {res }}^{A P}} \sum_{k_{\tau}=1}^{24} \sum_{i=1}^{N_{\mathrm{G}}} \sum_{j=1}^{N_{\mathrm{m}}} \frac{N_{u i}}{N_{\mathrm{m}} \cdot N_{\mathrm{p}}} \\
& \times \mu_{i j k_{\tau} k}^{A P} \cdot M_{i}^{A P}\left(P_{i k_{\tau}}\right), \\
\mathrm{AGLC}^{A P} & =\mathrm{TGLC}^{A P} /\left(24+k_{\mathrm{res}}^{A P}-1\right) .
\end{aligned}\right.
$$



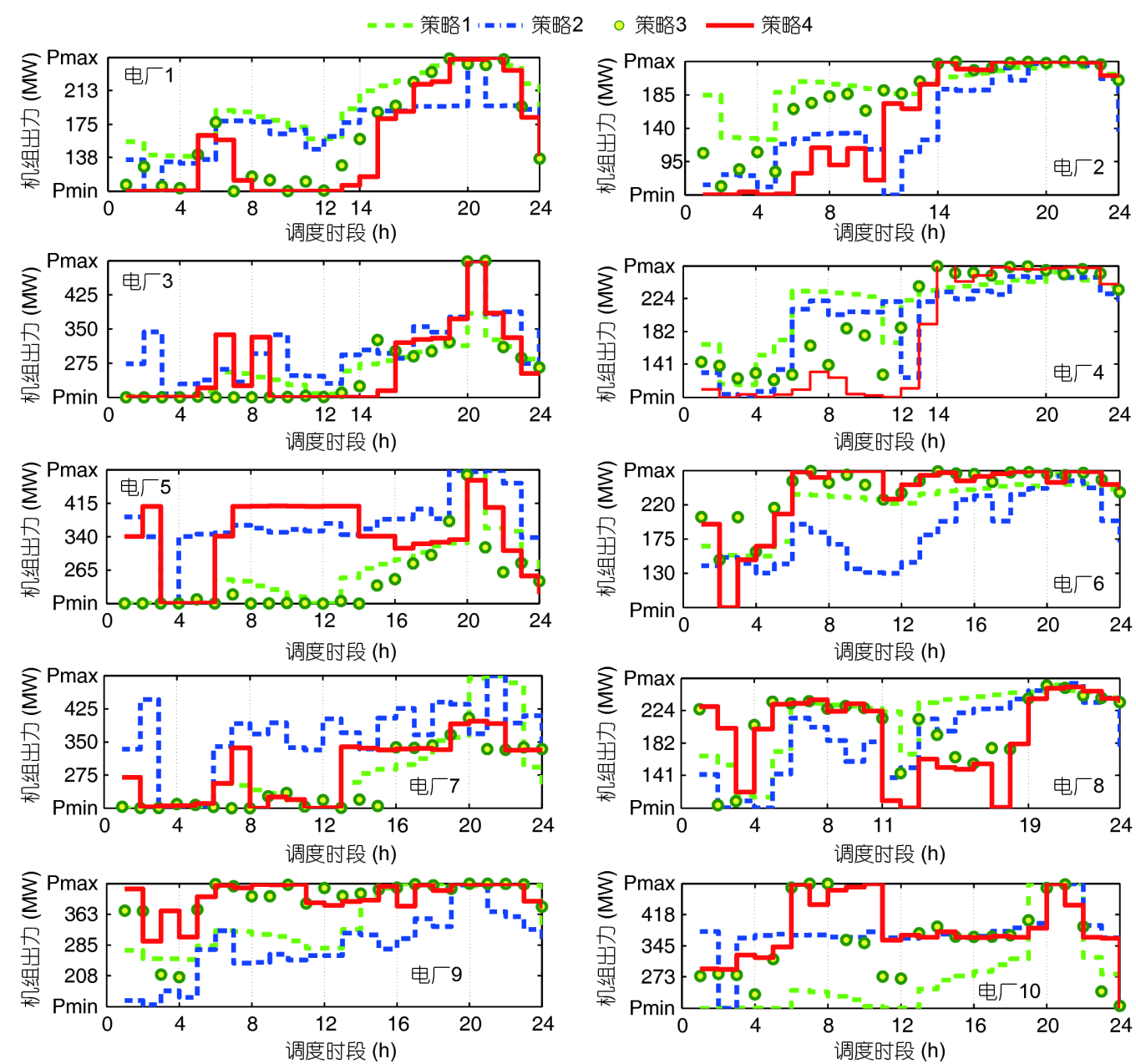

图 8 污染日场景四种策略的调度计划

表 4 模拟城市案例调度结果统计表

\begin{tabular}{|c|c|c|c|c|c|c|c|c|c|c|c|c|}
\hline \multirow{2}{*}{ 气象场景 } & \multirow{2}{*}{$\begin{array}{l}\text { 调度 } \\
\text { 策略 }\end{array}$} & \multirow{2}{*}{$\begin{array}{c}\text { TGC } \\
(\$)\end{array}$} & \multirow{2}{*}{$\begin{array}{c}\text { TEC } \\
\left(10^{4} t\right)\end{array}$} & \multicolumn{3}{|c|}{$\mathrm{PM}_{2.5}$} & \multicolumn{3}{|c|}{$\mathrm{SO}_{2}$} & \multicolumn{3}{|c|}{$\mathrm{NO}_{2}$} \\
\hline & & & & $\begin{array}{l}\mathrm{TE} \\
(\mathrm{t})\end{array}$ & $\begin{array}{c}\text { TGLC } \\
\left(\mu \mathrm{g} / \mathrm{m}^{3}\right)\end{array}$ & $\begin{array}{l}\text { AGLC } \\
\left(\mu \mathrm{g} / \mathrm{m}^{3}\right)\end{array}$ & $\begin{array}{l}\mathrm{TE} \\
(\mathrm{t})\end{array}$ & $\begin{array}{c}\text { TGLC } \\
\left(\mu \mathrm{g} / \mathrm{m}^{3}\right)\end{array}$ & $\begin{array}{l}\text { AGLC } \\
\left(\mu \mathrm{g} / \mathrm{m}^{3}\right)\end{array}$ & $\begin{array}{l}\mathrm{TE} \\
(\mathrm{t})\end{array}$ & $\begin{array}{c}\text { TGLC } \\
\left(\mu \mathrm{g} / \mathrm{m}^{3}\right)\end{array}$ & $\begin{array}{l}\text { AGLC } \\
\left(\mu \mathrm{g} / \mathrm{m}^{3}\right)\end{array}$ \\
\hline \multirow{4}{*}{ 污染日 } & 策略1 & 22069.36 & 10.00 & 36.97 & 50.99 & 1.13 & 89.18 & 134.42 & 2.86 & 260.34 & 357.11 & 8.30 \\
\hline & 策略2 & 24856.43 & 10.15 & 30.83 & 45.07 & 1.00 & 70.04 & 100.61 & 2.14 & 202.31 & 293.68 & 6.83 \\
\hline & 策略3 & 24013.06 & 9.97 & 36.21 & 38.30 & 0.85 & 87.31 & 110.39 & 2.35 & 258.04 & 270.83 & 6.30 \\
\hline & 策略4 & 25388.95 & 10.04 & 34.32 & 30.83 & 0.69 & 82.37 & 89.29 & 1.90 & 242.96 & 214.90 & 5.00 \\
\hline \multirow{4}{*}{ 非污染日 } & 策略1 & 22069.36 & 10.00 & 36.97 & 1.90 & 0.04 & 89.18 & 4.23 & 0.09 & 260.34 & 12.38 & 0.28 \\
\hline & 策略2 & 24856.43 & 10.15 & 30.83 & 1.52 & 0.03 & 70.04 & 3.53 & 0.08 & 202.31 & 8.79 & 0.20 \\
\hline & 策略3 & 22933.60 & 9.94 & 37.43 & 1.04 & 0.02 & 91.19 & 2.64 & 0.06 & 264.86 & 6.42 & 0.14 \\
\hline & 策略4 & 23517.66 & 9.96 & 36.77 & 0.75 & 0.02 & 88.98 & 1.86 & 0.04 & 259.62 & 4.55 & 0.10 \\
\hline
\end{tabular}

可以看出：(1) 虽然策略 2 中 $\mathrm{PM}_{2.5}, \mathrm{SO}_{2}, \mathrm{NO}_{2}$ 的 TE 均为 4 种策略中最小, 但其 TGLC 却比策略 4 高出
$46.19 \%, 12.68 \%, 36.66 \%$, 而GLC才是衡量大气污染物 危害的真正标准，因此排放量限制法并非减少火电厂 
大气污染的有效手段; (2) 策略3中 $\mathrm{PM}_{2.5}$ 的TGLC虽比 策略 1 降低了 $24.9 \%$, 但其 $\mathrm{SO}_{2}$ 的 TGLC比策略 2 还高出 $9.72 \%, \mathrm{NO}_{2}$ 的TGLC比策略4高了 $26.03 \%$ ，说明了仅对 一种污染物进行控制就欲实现空气质量的全面改善是 不可行的，应结合各污染物的特点进行分别、同时控 制;（3）相比而言，策略4中3种大气污染物的TGLC及 AGLC均为所有策略中的最小, 且 TGC仅比策略2高 $2.10 \%$, TEC 也降低了 $1.08 \%$, 具有明显的优势. 值得指 出的是, 此策略中 $\mathrm{SO}_{2}$ 的优化力度小于 $\mathrm{PM}_{2.5}$ 和 $\mathrm{NO}_{2}$ 的优 化力度, 这是因为当日 $\mathrm{SO}_{2}$ 的IAQI始终处于“优”和“良” 区间内, 其环境容量较大, 在求取折中解时的目标权重 值较小. 说明了策略4可以根据环境容量对不同污染物 进行差别化调控. 决策者亦可根据实际调度准则及自 身经验在各时段的帕累托解集中选择满意解，灵活地 使电厂出力达到某一非劣状态.

为了排除目标权值带来的影响，更好地说明本文 所提出的以最小化EAQ为目标的火电厂大气污染控 制方法相对于传统的排放量限制法的优势，本文进一 步以 $[0,0,1,0,0],[0,0,0,1,0],[0,0,0,0,1]$ 为目标权重在策 略 2 和 4 所得 $\mathrm{PF}$ 上篮选解，从而得到两策略分别针对减 轻 $\mathrm{PM}_{2.5}, \mathrm{SO}_{2}, \mathrm{NO}_{2}$ 的大气污染的极端解, 结果如表 5 所 示. 可以看出, 在 3 种极端权重方案中, 虽策略 2 的各污 染物排放量均为最低，但策略4中 $\mathrm{PM}_{2.5}, \mathrm{SO}_{2}, \mathrm{NO}_{2}$ 的 AGLC却分别比策略2减小了 $18.75 \%, 14.43 \%, 18.81 \%$, 且 TGC 和TEC均小于策略2. 这充分说明了EAQ最小化 法确实是比传统的排放量限制法更有效的火电厂大气 污染控制方法, 通过将大气污染的时空分布考虑在内, $\mathrm{EAQ}$ 最小化法可以辨识出对大气污染真正有责任的 火电厂, 并采取针对性措施, 从而实现经济、高效的火 电厂大气污染控制.

\subsection{2 非污染日场景}

非污染日的气象要素数据如附录表 $\mathrm{S} 3$ 所示, 其特 征为: 风速较大 (最大至 $7.3 \mathrm{~m} / \mathrm{s}$ ), $z_{h}$ 较高(最大至 $1500 \mathrm{~m}$ ), 大气层结偏向于中性至不稳定, 有利于污染 物的扩散、稀释与输送，预测IAQI等级为“优”或“良”, 且当日该城市主导风为西南风, 大部分电厂都处于城 市的下风向, 对城市空气质量的影响较小.

仿真结果如表4所示. 由于策略 1 和 2 并不考虑大气 污染物的时空分布, 因此气象条件的变化对其调度结 果并无影响, 再加上两个场景下的负荷曲线完全相同, 故策略 1 和 2 在非污染日与污染日的调度结果也完全相 同. 对比这两个策略在不同场景下造成的GLC, 可以看 出: 即使在完全相同的排放量下, 气象条件的差异可使 $\mathrm{PM}_{2.5}, \mathrm{SO}_{2}, \mathrm{NO}_{2}$ 的 GLC 在非污染日比污染日低26 49 倍, 这充分说明了气象条件能对大气污染物的GLC产 生决定性影响, 进而证明了基于GLC展开火电厂大气 污染控制的必要性. 策略4不仅考虑了污染物的GLC, 还在挑选折中解时将气象条件变化所引起的环境容量 的改变考虑在内. 这使得策略4在空气质量状况较好、 大气污染治理压力较小的非污染日场景中, 灵活地把 优化重点转向了减少发电成本和降低碳排放, 调度计 划的经济性与碳减排特性明显增强: 与污染日相比, 在满足相同电力需求、空气质量合意的情况下, 此策 略在非污染日的TGC降低了1871.29美元、TEC降低 了 $0.08 \times 10^{4} \mathrm{t}$; 此外, 虽在污染日策略4因更侧重于缓解 大气污染而使其经济性比策略 2 略差, 但随着非污染日 大气污染治理压力的缓解, 策略 4 能充分发挥气象条件 和大气环境容量利用价值的特性使其在各方面都优于 策略2: 不仅 TGC, TEC减少了 1338.77 美元、 $0.19 \times 10^{4} \mathrm{t}$, $\mathrm{PM}_{2.5}, \mathrm{SO}_{2}, \mathrm{NO}_{2}$ 的AGLC也均更小, 具有十分明显的

表 5 策略 2 和 4 极端解结果分析

\begin{tabular}{|c|c|c|c|c|c|c|c|c|c|c|c|c|c|}
\hline \multirow[b]{2}{*}{ 目标权重 } & \multirow{2}{*}{$\begin{array}{l}\text { 调度 } \\
\text { 策略 }\end{array}$} & \multirow{2}{*}{$\begin{array}{c}\text { 极端解 } \\
\text { 对应的 } \\
\text { 目标 }\end{array}$} & \multirow[b]{2}{*}{$\begin{array}{c}\text { TGC } \\
(\$)\end{array}$} & \multirow{2}{*}{$\begin{array}{c}\text { TEC } \\
\left(10^{4} \mathrm{t}\right)\end{array}$} & \multicolumn{3}{|c|}{$\mathrm{PM}_{2.5}$} & \multicolumn{3}{|c|}{$\mathrm{SO}_{2}$} & \multicolumn{3}{|c|}{$\mathrm{NO}_{2}$} \\
\hline & & & & & $\begin{array}{l}\text { TE } \\
(\mathrm{t})\end{array}$ & $\begin{array}{c}\text { TGLC } \\
\left(\mu \mathrm{g} / \mathrm{m}^{3}\right)\end{array}$ & $\begin{array}{c}\text { AGLC } \\
\left(\mu \mathrm{g} / \mathrm{m}^{3}\right)\end{array}$ & $\begin{array}{l}\mathrm{TE} \\
(\mathrm{t})\end{array}$ & $\begin{array}{c}\text { TGLC } \\
\left(\mu \mathrm{g} / \mathrm{m}^{3}\right)\end{array}$ & $\begin{array}{l}\text { AGLC } \\
\left(\mu \mathrm{g} / \mathrm{m}^{3}\right)\end{array}$ & $\begin{array}{l}\mathrm{TE} \\
(\mathrm{t})\end{array}$ & $\begin{array}{c}\text { TGLC } \\
\left(\mu \mathrm{g} / \mathrm{m}^{3}\right)\end{array}$ & $\begin{array}{c}\text { AGLC } \\
\left(\mu \mathrm{g} / \mathrm{m}^{3}\right)\end{array}$ \\
\hline \multirow{2}{*}[0,0,1,0,0]{} & 策略2 & $\mathrm{E}^{\mathrm{PM} 2.5}$ & 29520.92 & 10.33 & 25.94 & 36.01 & 0.80 & 69.66 & 103.19 & 2.20 & 162.33 & 231.98 & 5.39 \\
\hline & 策略4 & $\mathrm{EAQ}^{\mathrm{PM} 2.5}$ & 27471.16 & 10.13 & 32.52 & 29.25 & 0.65 & 77.06 & 85.36 & 1.82 & 225.98 & 196.18 & 4.56 \\
\hline \multirow{2}{*}[0,0,0,1,0]{} & 策略2 & $\mathrm{E}^{\mathrm{SO} 2}$ & 28669.07 & 10.25 & 29.17 & 41.88 & 0.93 & 65.14 & 94.52 & 2.01 & 189.12 & 263.98 & 6.14 \\
\hline & 策略4 & $\mathrm{EAQ}^{\mathrm{SO} 2}$ & 27601.71 & 10.11 & 33.25 & 31.66 & 0.70 & 76.38 & 80.82 & 1.72 & 229.25 & 196.44 & 4.57 \\
\hline \multirow{2}{*}[0,0,0,0,1]{} & 策略2 & $\mathrm{E}^{\mathrm{NO} 2}$ & 29377.11 & 10.33 & 26.20 & 36.39 & 0.81 & 71.16 & 103.84 & 2.21 & 160.41 & 230.88 & 5.37 \\
\hline & 策略4 & $\mathrm{EAQ}^{\mathrm{NO2}}$ & 27564.57 & 10.14 & 32.48 & 29.85 & 0.66 & 77.76 & 87.06 & 1.85 & 222.83 & 187.50 & 4.36 \\
\hline
\end{tabular}


优势.

结合以上两个场景的仿真结果可以看出, 策略4不 仅可以高效改善空气质量，还可以根据环境容量及气 象条件灵活调整控制力度，有针对性地对重点污染物 进行控制, 实现了经济性、环保性的有机统一.

\section{4 广东省案例}

本节根据广东省实际的气象数据(http://data.cma. $\mathrm{cn} / \mathrm{site} / \mathrm{index} . \mathrm{html}$ )及全省 104 个空气质量监测站发布的 IAQI数据(http://beijingair.sinaapp.com), 对某一典型日 全省范围内的59家火电厂、165台机组制定调度计划, 其中, 煤电机组 134 台, 气电机组 31 台, 总装机容量 $61838 \mathrm{MW}$ ，同一电厂的同类机组间的出力仍均匀分 配, 负荷曲线见图9.

4种策略的调度结果如表6所示. 可以看出, 策略4 的各污染物TGLC和AGLC仍均为最小, 与策略 2 相比, 策略4不仅在减少大气污染方面更直接有效，还可减 少 56.05 万美元的 TGC. 火电厂并非唯一的大气污染 源, 欲通过其一家之力就实现全省范围内空气质量的 彻底改善几乎是不可能的，但策略 4 能够在保证电力 供应的基础上尽火电厂最大之力减少发电带来的环境 影响，各污染物TGLC可比策略1降低11.28\%, 10.01\%, $13.75 \%$. 虽直接成本投入增加了 373.64 万美元，但带来 的社会效益是相当可观的.

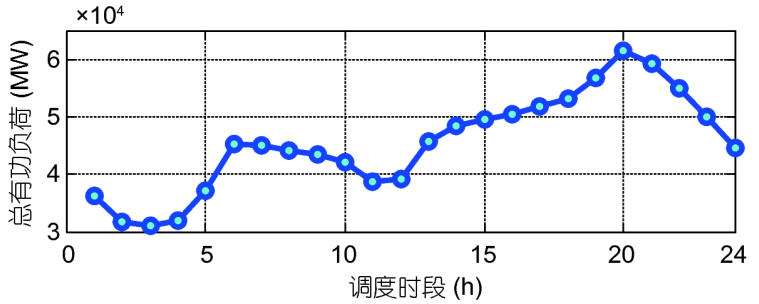

图 9 广东省案例负荷曲线

区域尺度下环境容量的空间差异较城市尺度更为 明显, 故本文进一步在此案例中验证策略4对不同城市 空气质量的差别化调控能力. 以19:00策略1的各城市的 $\mathrm{PM}_{2.5}$ 地面浓度为 1 (p.u.), 各调度策略结果对比如图 10 所示. 可以看出, 策略4能够对污染严重的城市进行重点 优化, GLC减少幅度比策略2和3明显加大, 如对佛山、 清远、肇庆; 而对于环境容量较大的城市，策略4又能 够灵活地弱化控制力度, 如对珠海、中山. 相比而言, 策略2不考虑环境的自净能力而只一味地控制排放量, 直接导致管理力度不够(如对清远、肇庆、江门、东 莞)或过度管控(如对惠州). 综上所述, 策略 4 是比排放 量限制法更具实用价值的经济、环保电力调度方法.

\section{6 结论}

提出了一种考虑多种污染物时空分布的电力系统 高维多目标优化调度策略, 主要包括以下贡献.

表 6 广东省案例调度结果统计表

\begin{tabular}{|c|c|c|c|c|c|c|c|c|c|c|c|}
\hline \multirow[b]{2}{*}{ 调度策略 } & \multirow{2}{*}{$\begin{array}{c}\text { TGC } \\
\left(10^{4} \$\right)\end{array}$} & \multirow{2}{*}{$\begin{array}{l}\text { TEC } \\
\left(10^{4} \mathrm{t}\right)\end{array}$} & \multicolumn{3}{|c|}{$\mathrm{PM}_{2.5}$} & \multicolumn{3}{|c|}{$\mathrm{SO}_{2}$} & \multicolumn{3}{|c|}{$\mathrm{NO}_{2}$} \\
\hline & & & $\begin{array}{l}\mathrm{TE} \\
(\mathrm{t})\end{array}$ & $\begin{array}{l}\text { TGLC } \\
\left(\mu \mathrm{g} / \mathrm{m}^{3}\right)\end{array}$ & $\begin{array}{l}\text { AGLC } \\
\left(\mu \mathrm{g} / \mathrm{m}^{3}\right)\end{array}$ & $\begin{array}{l}\mathrm{TE} \\
(\mathrm{t})\end{array}$ & $\begin{array}{l}\text { TGLC } \\
\left(\mu \mathrm{g} / \mathrm{m}^{3}\right)\end{array}$ & $\begin{array}{l}\text { AGLC } \\
\left(\mu \mathrm{g} / \mathrm{m}^{3}\right)\end{array}$ & $\begin{array}{l}\mathrm{TE} \\
(\mathrm{t})\end{array}$ & $\begin{array}{l}\text { TGLC } \\
\left(\mu \mathrm{g} / \mathrm{m}^{3}\right)\end{array}$ & $\begin{array}{l}\text { AGLC } \\
\left(\mu \mathrm{g} / \mathrm{m}^{3}\right)\end{array}$ \\
\hline 策略1 & 4260.97 & 90.42 & 376.13 & 98.74 & 2.19 & 1172.09 & 271.48 & 5.78 & 2013.00 & 561.07 & 13.05 \\
\hline 策略2 & 4690.66 & 88.05 & 350.50 & 91.37 & 2.03 & 1105.69 & 255.87 & 5.44 & 1850.16 & 513.31 & 11.94 \\
\hline 策略3 & 4672.22 & 88.43 & 361.99 & 90.58 & 2.01 & 1155.97 & 252.68 & 5.38 & 1886.49 & 513.99 & 11.95 \\
\hline 策略4 & 4634.61 & 88.34 & 358.44 & 87.60 & 1.95 & 1144.20 & 244.31 & 5.20 & 1865.91 & 483.92 & 11.25 \\
\hline
\end{tabular}

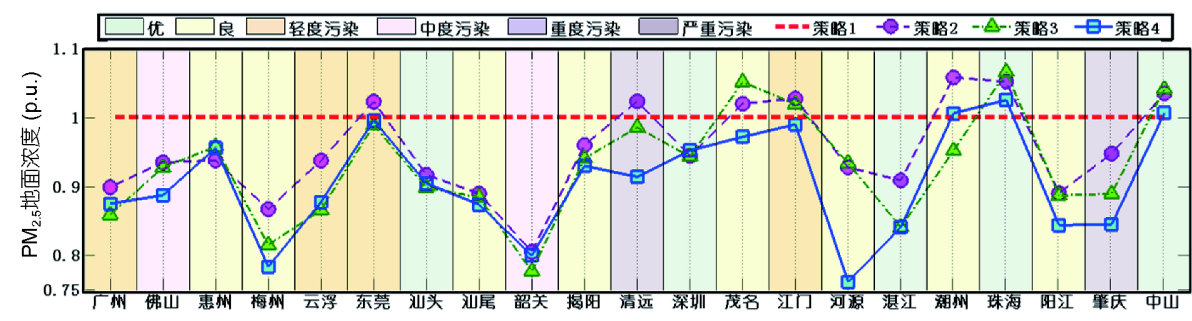

图 10 广东省21个城市各策略19:00 $\mathrm{PM}_{2.5}$ 地面浓度结果对比 
1) 构建出一种真正适用于电力调度的火电厂时 空分布模型，不仅可以用于各种气象条件、计算简便， 还综合考虑了连续烟流的精确模拟、ABL日变化及污 染物的影响寿命，充分体现了火电厂这类高架点源的 污染物扩散特征, 结果准确.

2) 建立了全新的考虑多种污染物时空分布的电 力系统高维多目标优化调度模型，分别从排放量和 $\mathrm{GLC}$ 的角度对 $\mathrm{CO}_{2}$ 及大气污染物进行管控, 比现行的 排放量限制法更直接有效，可在污染日使 $\mathrm{PM}_{2.5}, \mathrm{SO}_{2}$, $\mathrm{NO}_{2}$ 的GLC多降低 $31.60 \%, 11.25 \%, 26.83 \%$, 并能根据 不同城市、不同污染物的情况展开差别化调控.

3) 提出了一种考虑目标特征与环境容量的多目
标决策方法，可根据气象条件的时空变化对调度策略 做出灵活调整。在非污染日比排放量限制法多节省 1338.77 美元、多降低碳排放 $0.19 \times 10^{4} \mathrm{t} ，$ 大气污染治 理效果也更优，真正意义上实现经济、环保的电力 调度.

所提出的火电厂的污染物时空分布模型及EAQ函 数, 最终通过系数 $\pi$ 来计算, 可以很方便地与其他形式 的经济调度模型相结合, 如光滑二次型的经济调度模 型、考虑了阀点效应与禁止运行区间的经济调度模型 等,进而实现更高效的环境经济调度; 还可以很方便地 扩展到电力系统的其他优化问题中，如机组组合、综 合能源调度等, 具有广阔的应用前景.

\section{补充材料}

本文的补充材料见网络版techen.scichina.com. 补充材料为作者提供的原始数据, 作者对其学术质量和内容负责.

\section{参考文献}

1 郑楚光, 赵永椿, 郭欣. 中国富氧燃烧技术研发进展. 中国电机工程学报, 2014, 34: 3856-3864

2 Yang B, Yu T, Shu H, et al. Robust sliding-mode control of wind energy conversion systems for optimal power extraction via nonlinear perturbation observers. Appl Energy, 2018, 210: 711-723

3 Yang B, Zhang X, Yu T, et al. Grouped grey wolf optimizer for maximum power point tracking of doubly-fed induction generator based wind turbine. Energy Convers Manage, 2017, 133: 427-443

4 Yang B, Yu T, Shu H, et al. Democratic joint operations algorithm for optimal power extraction of PMSG based wind energy conversion system. Energy Convers Manage, 2018, 159: 312-326

5 Wang S J, Shahidehpour S M, Kirschen D S, et al. Short-term generation scheduling with transmission and environmental constraints using an augmented Lagrangian relaxation. IEEE Trans Power Syst, 1995, 10: 1294-1301

6 陈功贵, 陈金富. 含风电场电力系统环境经济动态调度建模与算法. 中国电机工程学报, 2013, 33: 27-35

7 Abido M A. A novel multiobjective evolutionary algorithm for environmental/economic power dispatch. Electric Power Syst Res, 2003, 65: 7181

8 Abido M A. Multiobjective particle swarm optimization for environmental/economic dispatch problem. Electric Power Syst Res, 2009, 79: 11051113

9 Hota P K, Barisal A K, Chakrabarti R. Economic emission load dispatch through fuzzy based bacterial foraging algorithm. Int J Electrical Power Energy Syst, 2010, 32: 794-803

10 Mandal K K, Mandal S, Bhattacharya B, et al. Non-convex emission constrained economic dispatch using a new self-adaptive particle swarm optimization technique. Appl Soft Comput, 2015, 28: 188-195

11 Balachandar P, Ganesan S, Jayakumar N , et al. Multi-fuel power dispatch in an interconnected power system using ant lion optimizer. Int J Energy Opt Eng, 2017, 6: 29-54

12 Yalcinoz T, Koksoy O. A multiobjective optimization method to environmental economic dispatch. Int J Electrical Power Energy Syst, 2007, 29: $42-50$

13 吴兑. 温室气体与温室效应. 北京: 气象出版社, 2003

14 国家环境保护总局. 环境空气质量监测规范(试行). 北京: 国家环境保护总局, 2007

15 国家环境保护总局. 环境空气质量指数(AQI)技术规定(试行) (HJ 633-2012). 北京: 中国环境科学出版社, 2012 
16 Lei S, Hou Y, Wang X, et al. Unit commitment incorporating spatial distribution control of air pollutant dispersion. IEEE Trans Ind Inf, 2017, 13: 995-1005

17 Sharan M, Yadav A K, Singh M P, et al. A mathematical model for the dispersion of air pollutants in low wind conditions. Atmos Environ, 1996, 30: $1209-1220$

18 Chu K C, Jamshidi M, Levitan R. An approach to on-line power dispatch with ambient air pollution constraints. IEEE Trans Automat Contr, 1977, 22: 385-396

19 蒋维楣. 空气污染气象学教程. 北京: 气象出版社, 2004

20 国家技术监督局, 国家环境保护总局. 制定地方大气污染排放标准的技术方法(GB/T 3840-91). 北京: 中国标准出版社, 1992

21 Stull R B. An Introduction to Boundary Layer Meteorology. London: Kluwer Academic Publishers, 1988

Kaimal J C, Finnigan J J. Atmospheric Boundary Layer Flows Their Structure and Measurement. New York: Oxford University Press, 1994. 1-10

3 林伟. 火力发电厂烟图高度影响因素的初步分析. 电力勘测设计, 2013, 5: 46-51

24 Paolo Z. Air Pollution Modeling. New York: Springer Science + Business Media, 1990. 153-154

25 Hunt J C R. Diffusion in the stable boundary layer. In: Nieuwstadt F T M, van Dop H, eds. Atmospheric Turbulence and Air Pollution Modelling. Dordrecht: Springer, 1984. 231-274

26 Ludwig F L, Gasiorek L S, Ruff R E. Simplification of a gaussian puff model for real-time minicomputer use. Atmos Environ, 1977, 11: 431-436

27 Zannetti P, Al-Madani N. Simulation of transformation, buoyancy and removal processes by Lagrangian Particle Methods. In: De Wispelaere C, ed. Air Pollution Modeling and Its Application IV. Nato-Challenges of Modern Society (Energy Engineering and Advanced Power Systems), vol 7. Boston: Springer, 1985

28 Lin C, Viviani G. Hierarchical economic dispatch for piecewise quadratic cost functions. IEEE Trans Power Apparatus Syst, 1984, PAS-103: $1170-1175$

29 詹俊鹏, 郭创新, 吴青华, 等. 快速群搜索优化算法及其在电力系统经济调度中的应用. 中国电机工程学报, 2012, 32: 1-6

30 Binetti G, Davoudi A, Naso D, et al. A distributed auction-based algorithm for the nonconvex economic dispatch problem. IEEE Trans Ind Inf, 2014, 10: 1124-1132

31 Chiang C L. Improved genetic algorithm for power economic dispatch of units with valve-point effects and multiple fuels. IEEE Trans Power Syst, 2005, 20: 1690-1699

32 Thang N T. Economic emission load dispatch with multiple fuel options using hopfield Lagrange network. Int J Adv Sci Technol, 2014, 57: 9-24 马小姝, 李宇龙, 严浪. 传统多目标优化方法和多目标遗传算法的比较综述. 电气传动自动化, 2010, 32: 48-50

36 Deb K, Jain H. An evolutionary many-objective optimization algorithm using reference-point-based nondominated sorting approach, Part I: Solving problems with box constraints. IEEE Trans Evol Comput, 2014, 18: 577-601 Yang S, Li M, Liu X, et al. A grid-based evolutionary algorithm for many-objective optimization. IEEE Trans Evol Computat, 2013, 17: 721-736 Zitzler E, Künzli S. Indicator-based selection in multiobjective search. In: Parallel Problem Solving from Nature (PPSN VIII). Berlin: Springer, 2004. $832-842$

39 Zhang Q F, Li H. MOEA/D: A multiobjective evolutionary algorithm based on decomposition. IEEE Trans Evol Comput, 2007, 11: 712-731

40 Diakoulaki D, Mavrotas G, Papayannakis L. Determining objective weights in multiple criteria problems: The critic method. Comput Operat Res, 1995, 22: 763-770

41 王中兴, 李桥兴. 依据主客观权重集成最终权重的一种方法. 应用数学与计算数学学报, 2006, 20: 87-92

42 袁亚湘. 非线性规划数值方法. 上海: 上海科学技术出版社, 1993

43 陈雷, 王延章. 基于熵权系数与TOPSIS集成评价决策方法的研究. 控制与决策, 2003, 18: 456-459

44 桑建国. 大气扩散的数值计算. 北京: 气象出版社, 1992

45 郁否. MATLAB智能算法 20 个案例分析. 北京: 航空航天大学出版社, 2011

46 He S, Wu Q H, Saunders J R. Group search optimizer: An optimization algorithm inspired by animal searching behavior. IEEE Trans Evol Comput, 2009, 13: 973-990

47 周斌, 宋艳, 李金茗, 等. 基于多群组均衡协同搜索的多目标优化发电调度. 电工技术学报, 2015, 30: 181-189 


\title{
Many-objective optimization dispatching strategy for power system considering the temporal and spatial distribution of different pollutants
}

\author{
YU Tao, CHEN YiXuan \& ZHANG XiaoShun \\ School of Electrical Power, South China University of Technology, Guangzhou 510640, China
}

A novel many-objective optimization dispatching strategy for power system considering the temporal and spatial distribution of different pollutants is proposed in this paper. Firstly, a thermal power plant pollutant temporal and spatial distribution model is constructed which is truly suitable for power dispatching. The diurnal variation of atmospheric boundary layer is taken into account for the first time, reflecting the pollutant dispersion characteristics of power plants and improving the accuracy. Then, combined with the temporal and spatial distribution characteristics of various pollutants and environmental capacity, a many-objective power dispatching model is constructed to reduce the generation cost, carbon emissions and the effects on air quality by $\mathrm{PM}_{2.5}, \mathrm{SO}_{2}$ and $\mathrm{NO}_{2}$. The approximate Pareto optimal set is obtained by representative algorithms and a multi-objective decision method considering the features of different objectives and environmental capacity is proposed. The simulation results of the simulated city and Guangdong shows that the dispatching method can not only effectively improve air quality, but also be adjusted according to the environmental capacity, realizing the economic and environmental power dispatching in real sense.

atmospheric boundary layer, power plant pollutant temporal and spatial distribution model, many-objective optimization problem, power dispatching, environmental capacity

doi: $10.1360 /$ N092017-00355 